\title{
New methods for interaction analysis of complex processes using weighted graphs
}

\author{
Miguel Castaño Arranz*, Wolfgang Birk \\ Luleå University of Technology, SE-971 87 Luleå, Sweden
}

\section{A R T I C L E I N F O}

\section{Article history:}

Received 14 January 2011

Received in revised form 9 June 2011

Accepted 23 July 2011

Available online 19 September 2011

\section{Keywords:}

Complex systems

Interaction measures

Structural properties

System analysis

\begin{abstract}
A B S T R A C T
The selection of the structure of a controller in large scale industry processes usually requires extensive process knowledge. The aim of this paper is to report new results on recently suggested methods for the analysis of complex processes. These methods aid the designers in comprehending a process by representing structural and functional relationships from actuators and process disturbances to measured or estimated variables. The methods are formulated in a flexible framework based on graph theory, which can also be used for closed-loop analysis. Additionally, the sensitivity of the methods to scaling and time delays are discussed and resolved. It is also proposed how filtering can be used to restrict the analysis to a frequency region of interest.

The feasibility of the methods is shown by the use of three case studies. A quadruple tank process is used to exemplify the methods and their use. Then the methods are applied on a real-life process, the stock preparation plant of a pulp and paper mill. The third study case analyzes a previously published example in closed loop.

It is shown that the methods can be used to take efficient decisions on decentralized and sparse control structures, as well as assessing the channel interactions in a closed-loop system.
\end{abstract}

(c) 2011 Elsevier Ltd. All rights reserved.

\section{Introduction}

Large scale industrial process plants are characterized by a high degree of interaction between process variables, where hundreds or even thousands of variables are connected through dynamic systems. Examples of such interconnections are material flows and reflows, the latter e.g. due to discarded material being returned to previous process steps which gives rise to large feedback loops. Other examples are connections through supply grids for e.g. pressurized air. One process step consuming pressurized air may give rise to a pressure drop that propagates to every other consumer in the plant. Adding control loops to the process on different levels of hierarchy may result in a system with unintelligible causality and unpredictable dynamics.

For the control engineer, these very complex interconnected systems are a challenge. The question is how to represent the complexity in a comprehensible way and how to analyze it regarding e.g. dynamic behavior and control structure design. Traditionally, interaction measures are used for the control structure design, namely control structure selection and decision on the controller configuration. Control structure selection is about determining which dynamic interconnections should be used for the controller design;

\footnotetext{
* Corresponding author. Tel.: +46920492328.

E-mail address: miguel.castano@ltu.se (M. Castaño Arranz).
}

it is also referred to as input/output selection, see e.g. [1]. When the structure is selected, the interconnections can be used to configure a controller, that is decisions on e.g. degree-of-freedom, feed-forwards, or cascaded configurations need to be taken. In this paper the focus will be on the controller structure selection.

One of the most widely spread control structures is the decentralized control structure in a one degree of freedom setting, and the most well-known methods to determine the interconnections that are used for the controller design are the Relative Gain Array (RGA) [2], and the Dynamic RGA [3]. An extension of control structure selection to block diagonal control structures is discussed in [4], [5]. Generally, the methods are designed from the perspective that all controlled and manipulated variables are considered during the structure selection and that the control configuration is often set a-priori. In the survey [1], most of the available methods for the control structure design are reviewed, apart from gramian based methods which were suggested more recently, [6,7]. The latter have the advantage that no assumption on the control configuration is used and virtually any control structure could be deduced. But their interpretation becomes difficult when the number of variables becomes large.

For large scale systems, where the amount of variables can be in the magnitude of thousands, usually the control structure selection need to be preceded by a step where manipulated and controlled variables are grouped into sets where the number of variables is reduced to a couple of dozens. Thus, making the above 
methods more applicable. Methods for such a decomposition are proposed in $[8,9]$, where state space representations are used and produce interconnected multivariable systems. It should also be noted that interconnected systems can also be represented by signal flow graphs, which dates back to the work of Mason [10], and provides a comprehensive visual interpretation at the same time.

An alternative to the above is the concept of plant wide control (PWC) which has received much more attention in recent years. Methodologies like the nine-step method proposed by Luyben et al. [11] or the self-optimizing control procedure by Skogestad [12] are suggested and are evaluated on realistic large scale system, see e.g. [13]. In this, a complete procedure to address the control problem of a large scale system is provided.

A shortcoming of the mentioned methods is either their inability to scale to large scale problems or that control structure and configuration are limited a-priori. The aim of this paper is to suggest new methods for the control structure selection without imposing limitations on the either structure or configuration. But for the time being, it is assumed that the large scale problem is already decomposed in multivariable problems with a rather limited number of manipulated and controlled variables. In order to demonstrate the usefulness of the suggested methods, a $2 \times 2$ system with disturbances (Quadruple tank laboratory setup) and a $5 \times 5$ real-life process (Stock preparation plant at SCA Obbola AB, Sweden) are discussed.

The methods are inspired by the work within brain connectivity theory [14], where signal flow graphs can be analyzed from the perspective of the signal energy or power that is transferred from a selected input/output set of nodes. In this context, structural and functional properties are treated separately, where the structural properties capture the structural nature of a signal flow graph, while the functional properties resolve all intermediate connections in order to map the properties to the input/output set alone. Consequently, the analysis can be adapted to the needs for the task at hand, which makes the methods more versatile.

As a result quantitative methods are combined with the signal flow graphs approach by introducing weights on the edges. It will be shown that the $\mathcal{H}_{2}$-norm can be effectively used to assess the significance of edges in relation to each other, namely by the assessment of the transfer of signal energy or power. It is the authors believe that this combination enables control engineers to make better decisions on both control structure selection and controller configuration. But, it still remains to prove the scalability to large scale systems and if the methods can be used in the scope of the previously mentioned PWC procedures. In a preparatory step and to facilitate applicability the methods have been integrated into a prototype tool called ProMoVis, that can be used to both model, visualize and analyze industrial processes, [15].

The paper layout is as follows. First the preliminaries for the representation of linear systems as a signal flow graph is given in Section 2. Section 3 introduces a methodology for obtaining a scaling independent representation of linear processes as a signal flow graph. Section 4 describes the quadruple tank process and derives a scaling independent representation for a selected working point. Section 5 describes the norms and normalizations which will be used for quantifying and comparing the significance of the process interconnections. The quadruple tank process is used in Section 6 as an illustrative example for introducing the methods for the structural and functional analysis of complex processes which are the subject of this paper. Section 7 describes how to interpret the methods and use them for control structure selection, comparing the results obtained in the analysis of the quadruple tank with those of the RGA. Section 8 analyzes the scaling sensitivity of the methods for the cases in which a scaling independent representation of the process is not used. The stock preparation plant at SCA Obbola,
Sweden is a real-life process which is analyzed in this section to illustrate how the scaling issues present in the traditional gramian based IMs when analyzing industrial process can be resolved by using the discussed methods. Section 9 introduces the usefulness of the analysis methods for the evaluation of the interaction between control loops, and contains examples which show the use of filters for selecting a range of frequencies subject to analysis. The conclusions are finally given in Section 10.

\section{Signal flow graphs representing complex processes}

A representation of complex systems in relation to graph representations was introduced in [16]. The same representation will be used here dispensing with the output equation. When representing an open loop process, it will be considered that the input vector $u$ is composed by the exogenous inputs to the process (actions on actuators and process disturbances), and $x$ collects all the internal states and measurements which are subject to analysis, being both related in an expression like:

$x_{i}=\boldsymbol{\Phi}_{i 1} \cdot x_{1}+\cdots+\boldsymbol{\Phi}_{i n} \cdot x_{n}+\boldsymbol{\Gamma}_{i 1} \cdot u_{1}+\cdots+\boldsymbol{\Gamma}_{i p} \cdot u_{p}$

where $\boldsymbol{\Phi}_{i j}$ and $\boldsymbol{\Gamma}_{i j}$ are linear dynamic systems. The signal flow graph is then formulated as

$x=\boldsymbol{\Phi} x+\Gamma u$

Given a multivariable system $\boldsymbol{H}$, the duple $(\boldsymbol{\Phi}, \boldsymbol{\Gamma})$ is called a visualization of $\boldsymbol{H}$. The input-output matrix $\boldsymbol{\Omega}$ of $\boldsymbol{H}$ can be computed as $\boldsymbol{\Omega}=(I-\boldsymbol{\Phi})^{-1} \boldsymbol{\Gamma}$.

This formulation will also be used in Section 9 for representing a closed loop system. In that case, the reference signals for the closed loops are aggregated to the input vector $u$, and the control actions which are manipulated by a controller are now aggregated to the vector $x$ and removed from the input vector $u$.

The following transformations will be here used for obtaining process representations describing the nodes and interconnections which are of interest for the analysis.

\subsection{Hiding of self-references}

A node depending on itself is known as a self-reference. For the work described in this paper we will assume that the visualization representing the process has no self-references, that is, the diagonal of matrix $\boldsymbol{\Phi}$ is composed by zeros. If a visualization with self references is to be analyzed, the user has to first make an operation which hides the self-references and still preserves the physical structure of the plant. The details for such an operation are discussed in [16].

\subsection{Hiding arbitrary nodes}

Different levels of hierarchy are usually present when representing and analyzing large scale interconnected systems, and the hiding of nodes allows to disregard the variables which are not presently important while preserving the physical structure of the plant.

Consider the partitioning of the interconnected system $(\boldsymbol{\Phi}, \boldsymbol{\Gamma})$ as

$\left(\begin{array}{l}x_{1} \\ x_{2}\end{array}\right)=\left(\begin{array}{ll}\boldsymbol{\Phi}_{11} & \boldsymbol{\Phi}_{12} \\ \boldsymbol{\Phi}_{21} & \boldsymbol{\Phi}_{22}\end{array}\right)\left(\begin{array}{l}x_{1} \\ x_{2}\end{array}\right)+\left(\begin{array}{l}\boldsymbol{\Gamma}_{1} \\ \boldsymbol{\Gamma}_{2}\end{array}\right) u$

where $x_{2}=\left[\begin{array}{lll}0_{m \times(m-n)} & I_{m}\end{array}\right] \cdot x$ represents the last $m$ of the total $n$ nodes. Assuming that $x^{\prime}=\boldsymbol{\Phi}_{22} x^{\prime}+u^{\prime}$ is well-posed, then the 
visualization of the system when the last $m$ nodes are hidden, and which preserves the system structure is [16]:

$x_{1}=\hat{\boldsymbol{\Phi}} x_{1}+\hat{\boldsymbol{\Gamma}} u$

with

$\hat{\boldsymbol{\Phi}}=\boldsymbol{\Phi}_{11}+\boldsymbol{\Phi}_{12}\left(I-\boldsymbol{\Phi}_{22}\right)^{-1} \boldsymbol{\Phi}_{21} \quad, \quad \hat{\boldsymbol{\Gamma}}=\boldsymbol{\Gamma}_{1}+\boldsymbol{\Phi}_{12}\left(I-\boldsymbol{\Phi}_{22}\right)^{-1} \boldsymbol{\Gamma}_{2}$

Which can be expressed as a function $\mathcal{F}$ of $\boldsymbol{\Gamma}, \boldsymbol{\Phi}$, and $I_{m}$, being $m$ the number of nodes to be hidden.

$\left[\begin{array}{ll}\hat{\boldsymbol{\Gamma}} & \hat{\boldsymbol{\Phi}}\end{array}\right]=\left[\begin{array}{ll}\boldsymbol{\Gamma}_{1} & \boldsymbol{\Phi}_{11}\end{array}\right]+\boldsymbol{\Phi}_{12}\left(1-\boldsymbol{\Phi}_{22}\right)^{-1}\left[\begin{array}{ll}\boldsymbol{\Gamma}_{2} & \boldsymbol{\Phi}_{12}\end{array}\right]=\mathcal{F}\left(\left[\begin{array}{ll}\boldsymbol{\Gamma} & \boldsymbol{\Phi}\end{array}\right], I_{m}\right)$

To hide an arbitrary subset $\mathrm{N}$ of nodes, let us define the permutation matrix $T^{T}=\left[\begin{array}{ll}E^{T} & E^{\prime T}\end{array}\right]$ such that $x_{1}=E x$ is now a vector containing the nodes to be retained and $x_{2}=E^{\prime} x$ contains now the nodes to be hidden. The operation $\mathcal{H}_{N}$ of hiding an arbitrary set of nodes, is then:

$\mathcal{H}_{N}\left[\begin{array}{ll}\boldsymbol{\Gamma} & \boldsymbol{\Phi}\end{array}\right]=\mathcal{F}\left(T\left[\begin{array}{ll}\boldsymbol{\Gamma} & \boldsymbol{\Phi} \cdot T^{T}\end{array}\right], I_{m}\right)$

\section{Scaling independent representation}

Scaling is an important issue in many applications. The methods used in this paper are based on comparing the signal power (or energy) that can be transferred through the process interconnections. This power transfer is quantified as signal power transfer, and therefore it depends on the scales used to represent the analyzed signals.

Usual methods for scaling signals involve dividing each variable by its maximum expected or allowed change [17].

Let $\boldsymbol{Q}=(\boldsymbol{\Phi}, \boldsymbol{\Gamma})$ be a visualization of a system $\boldsymbol{H}$. When the input vector $u$ and the output vector $x$ are scaled by the diagonal scaling matrices $D_{u}$ and $D_{x}$ respectively, then the new input and output vectors are $\bar{u}=D_{u}^{-1} \cdot u$ and $\bar{x}=D_{x}^{-1} \cdot x$. The scaled visualization $\overline{\mathbf{Q}}=$ $(\overline{\boldsymbol{\Phi}}, \overline{\boldsymbol{\Gamma}})$ is then:

$\overline{\boldsymbol{\Phi}}=D_{x}^{-1} \boldsymbol{\Phi} D_{x} ; \quad \overline{\boldsymbol{\Gamma}}=D_{x}^{-1} \boldsymbol{\Gamma} D_{u}$

and the scaled input-output matrix $\overline{\boldsymbol{\Omega}}$ is:

$\overline{\mathbf{\Omega}}=D_{x}^{-1} \boldsymbol{\Omega} D_{u}$

It can be tedious to find an appropriate scaling for each of the process variables. Therefore, we present a representation of linear processes based on a visualization, which is independent of the selected scaling. For obtaining such a representation, an estimation of the standard deviation of the signals represented by the process variables is needed.

Let $\sigma_{u}, \sigma_{\bar{u}}, \sigma_{x}$ and $\sigma_{\bar{x}}$ be diagonal matrices collecting the standard deviation of each of the input or output signals and of their scaled version. Then, $\sigma_{\bar{u}}=\sigma_{u} D_{u}^{-1}$ and $\sigma_{\bar{x}}=\sigma_{x} D_{x}^{-1}$.

Lemma 1. The pair $\left(\boldsymbol{\sigma}_{x}^{-1} \boldsymbol{\Phi} \boldsymbol{\sigma}_{x}, \boldsymbol{\sigma}_{x}^{-1} \boldsymbol{\Gamma} \boldsymbol{\sigma}_{u}\right)$ is scaling invariant.

Proof.

$$
\begin{aligned}
\boldsymbol{\sigma}_{x}^{-1} \boldsymbol{\Phi} \boldsymbol{\sigma}_{x} & =\boldsymbol{\sigma}_{\bar{x}}^{-1} D_{x}^{-1} D_{x} \overline{\boldsymbol{\Phi}} D_{x}^{-1} D_{x} \boldsymbol{\sigma}_{\bar{x}}=\boldsymbol{\sigma}_{\bar{x}}^{-1} \overline{\boldsymbol{\Phi}} \boldsymbol{\sigma}_{\bar{x}} \\
\boldsymbol{\sigma}_{x}^{-1} \boldsymbol{\Gamma} \boldsymbol{\sigma}_{u} & =\sigma_{\bar{x}}^{-1} D_{x}^{-1} D_{x} \overline{\boldsymbol{\Gamma}} D_{u}^{-1} D_{u} \boldsymbol{\sigma}_{\bar{u}}=\boldsymbol{\sigma}_{\bar{x}}^{-1} \overline{\boldsymbol{\Gamma}} \boldsymbol{\sigma}_{\bar{u}}
\end{aligned}
$$

Given the visualization $\boldsymbol{Q}=(\boldsymbol{\Phi}, \boldsymbol{\Gamma})$ of a linear multivariable system $\boldsymbol{H}$, the pair $\tilde{\mathbf{Q}}=\left(\boldsymbol{\sigma}_{x}^{-1} \boldsymbol{\sigma}_{x}, \boldsymbol{\sigma}_{x}^{-1} \boldsymbol{\Gamma} \boldsymbol{\sigma}_{u}\right)$ will be named scaling invariant representation of $\boldsymbol{H}$.

Remark 1. The matrix $\tilde{\boldsymbol{\Omega}}=\left(I-\boldsymbol{\sigma}_{x}^{-1} \boldsymbol{\Phi} \boldsymbol{\sigma}_{x}\right)^{-1} \boldsymbol{\sigma}_{x}^{-1} \boldsymbol{\Gamma} \boldsymbol{\sigma}_{u}=\boldsymbol{\sigma}_{x}^{-1} \boldsymbol{\Omega} \boldsymbol{\sigma}_{u}$ related to the scaling invariant representation $\tilde{\mathbf{Q}}$, is also scaling invariant. This follows from:

$\boldsymbol{\sigma}_{\bar{x}}^{-1} \boldsymbol{\Omega} \boldsymbol{\sigma}_{u}=\boldsymbol{\sigma}_{\bar{x}}^{-1} D_{\bar{x}}^{-1} D_{x} \overline{\boldsymbol{\Omega}} D_{u}^{-1} D_{u} \boldsymbol{\sigma}_{\bar{u}}=\boldsymbol{\sigma}_{\bar{x}}^{-1} \overline{\boldsymbol{\Omega}} \boldsymbol{\sigma}_{\bar{u}}$
Remark 2. The premultiplication of matrix $\boldsymbol{\Omega}$ or $\boldsymbol{\Gamma}$ by $\boldsymbol{\sigma}_{x}^{-1}$ makes it invariant to output scaling, and the postmultiplication by $\sigma_{u}$ makes it invariant to input scaling. Matrix $\boldsymbol{\Phi}$ is independent of the selected input scaling, and the premultiplication by $\sigma_{x}^{-1}$ with the postmultiplication by $\sigma_{x}$ makes it independent to output scaling. Therefore, applying any of the structural or functional methods to the scaling invariant representation $\tilde{\mathbf{Q}}=\left(\boldsymbol{\sigma}_{x}^{-1} \boldsymbol{\Phi} \boldsymbol{\sigma}_{x}, \boldsymbol{\sigma}_{x}^{-1} \boldsymbol{\Gamma} \boldsymbol{\sigma}_{u}\right)$ with input-output matrix $\tilde{\boldsymbol{\Omega}}=\boldsymbol{\sigma}_{x}^{-1} \boldsymbol{\Omega} \boldsymbol{\sigma}_{u}$, always give the same result independently of the chosen scaling.

Remark 3. The gramian based IMs are sensitive to the scaling of the process variables $[7,6,18]$. The scaling invariant representation is also useful for computing a scaling invariant version of any of these IMs, by applying the corresponding method to the matrix $\tilde{\boldsymbol{\Omega}}=\boldsymbol{\sigma}_{x}^{-1} \boldsymbol{\Omega} \boldsymbol{\sigma}_{u}$.

The following section presents the quadruple tank process, and introduces an example of how to create a scaling independent representation of the process. Later, this representation will be used to apply the discussed structural and functional methods.

\section{The quadruple tank process}

The quadruple tank process has been introduced in [19], and is a well-known interacting process which has been used by a large number of authors as a benchmark to test several control and analysis methods. The process is depicted in Fig. 1. Two process disturbances have been added to the process and modeled as flow disturbances in the upper tanks. The differential equations of this modification were described in [20]. The process linear model can be formulated as a visualization as follows:
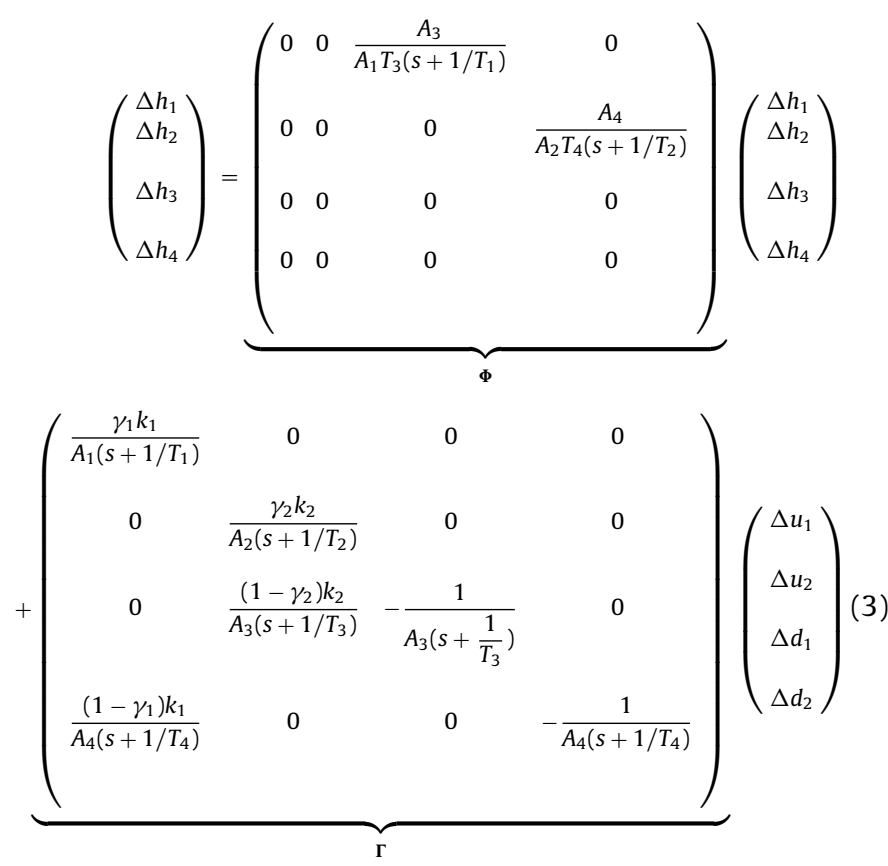

where $T_{i}$ are the time constants of the tanks

$T_{i}=\frac{A_{i}}{a_{i}} \sqrt{\frac{2 h_{i}^{o}}{g}}, \quad i=\{1,2,3,4\}$

The values of the construction parameters are given in Table 1.

The variables $u_{j}$ are associated with the two actuators and express the speed setting of the two pumps in \%. The measured 


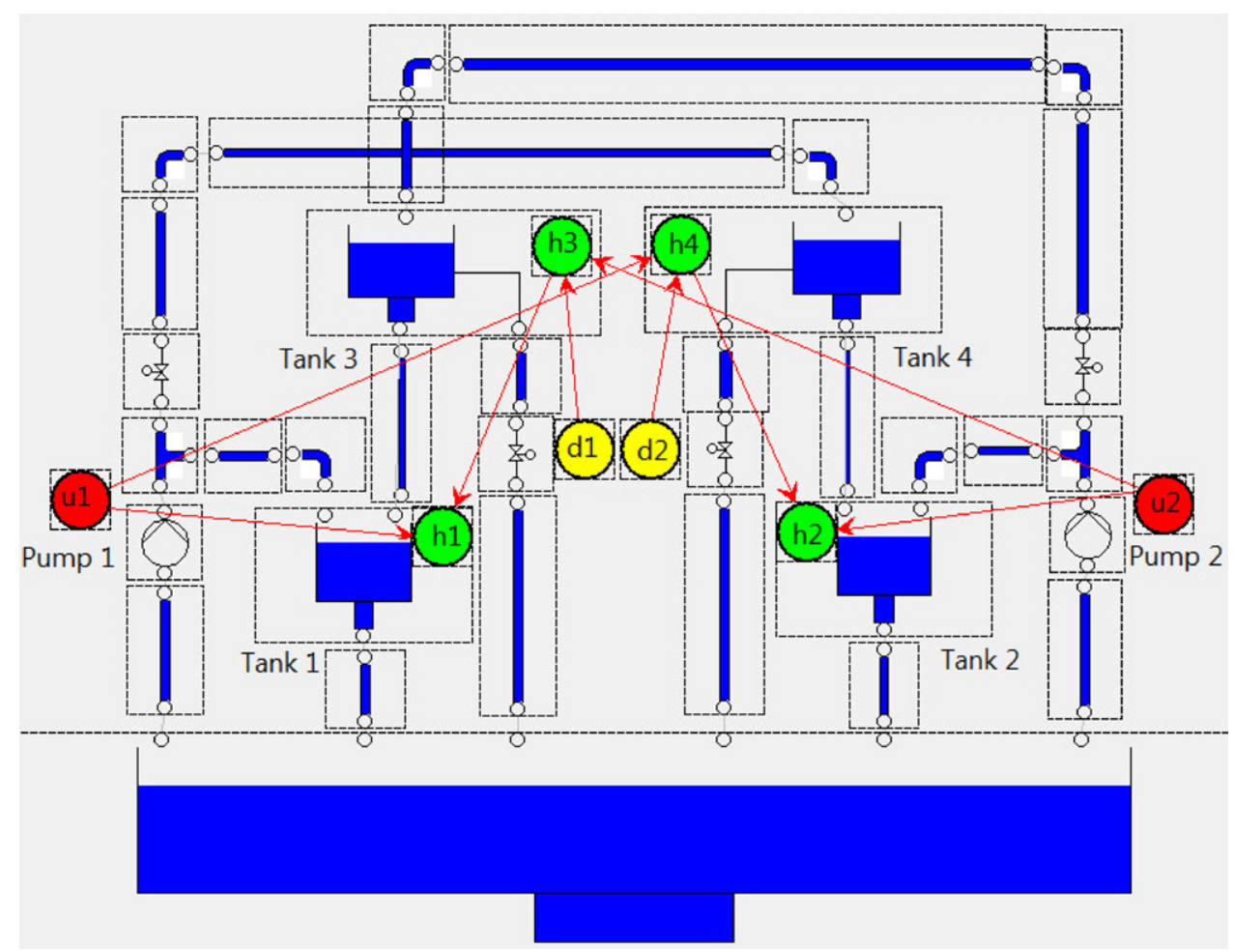

Fig. 1. Interacting system with water tanks. Each of the arrows represents a dynamic model connecting two process variables. ProMoVis screenshot

Table 1

Construction parameters of the quadruple tank process.

\begin{tabular}{|c|c|c|c|c|c|c|c|c|c|}
\hline & \multicolumn{9}{|l|}{ Parameter } \\
\hline & $A_{1}, A_{2}, A_{3}, A_{4}$ & $a_{1}$ & $a_{2}$ & $a_{3}$ & $a_{4}$ & $g$ & $k_{1}$ & $k_{2}$ & $\gamma_{1}, \gamma_{2}$ \\
\hline Value & $730 \mathrm{~cm}^{2}$ & $2.05 \mathrm{~cm}^{2}$ & $2.26 \mathrm{~cm}^{2}$ & $2.37 \mathrm{~cm}^{2}$ & $2.07 \mathrm{~cm}^{2}$ & $981 \mathrm{~cm} / \mathrm{s}^{2}$ & $7.45 \mathrm{~cm}^{3} /(\mathrm{s} \mathrm{V})$ & $7.30 \mathrm{~cm}^{3} /(\mathrm{sV})$ & 0.3 \\
\hline
\end{tabular}

Table 2

Selected working point for the quadruple tank process.

\begin{tabular}{|c|c|c|c|c|c|c|c|c|}
\hline & \multicolumn{8}{|c|}{ Variable } \\
\hline & $u_{1}$ & $u_{2}$ & $d_{1}$ & $d_{2}$ & $h_{1}$ & $h_{2}$ & $h_{3}$ & $h_{4}$ \\
\hline Value & 50 & 50 & 15.5 & 15.5 & 14.77 & 12.36 & 5.11 & 7 \\
\hline Range & $0-100$ & $0-100$ & $0-31$ & $0-31$ & $0-20$ & $0-20$ & $0-20$ & $0-20$ \\
\hline Units & $\%$ & $\%$ & $\mathrm{~cm}^{3} / \mathrm{s}$ & $\mathrm{cm}^{3} / \mathrm{s}$ & $\mathrm{cm}$ & $\mathrm{cm}$ & $\mathrm{cm}$ & $\mathrm{cm}$ \\
\hline
\end{tabular}

variables $h_{i}$ are the level of the tanks expressed in $\mathrm{cm}$. The two process disturbances $d_{1}$ and $d_{2}$ are flow perturbations in the top tanks, and are expressed in $\mathrm{cm}^{3} / \mathrm{s}$. The range of values which these variables can take is summarized in Table 2.

The input-output relationship is given by:
The working point selected for the analysis corresponds to an opening of $50 \%$ on both pumps, and the process disturbances are assumed to be at half of their possible maximum value. The values for the working point are summarized in Table 2 .

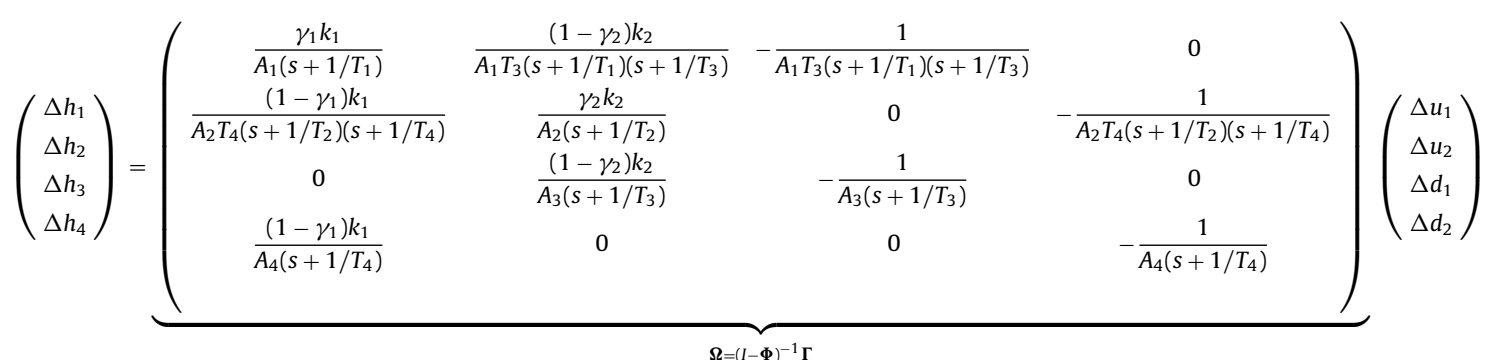


Example 1. The selection of the scaling factors for the process variables has an important impact on the analysis of the process interconnections. This selection is not trivial, as the scaling factors have to be chosen based on the expected variation of the process variables.

We proposed in the previous section a methodology for obtaining a system representation which is invariant with respect to the scaling of the process variables.

In order to demonstrate the utility of using the scaling invariant representation, the original system was first inappropriately scaled, so that the new input and output vectors, $\bar{u}$ and $\bar{y}$ are:

$\bar{u}=D_{u}^{-1} u \quad$ with $\quad D_{u}=\operatorname{diag}(100,1,15.5,15.5)$

$\bar{y}=D_{y}^{-1} y \quad$ with $\quad D_{y}=\operatorname{diag}(12.6472,1.9508,5.57,10.9376)$

The inputs $u_{1}$ and $u_{2}$ are scaled so their values are in the intervals $[0,1]$ and $[0 \%, 100 \%]$ respectively. The output levels which are originally expressed in $\mathrm{cm}$ are scaled with a randomly generated scaling matrix $D_{y}$. The process disturbances are scaled so that the allowed variation around the working point remains in the interval $[-1,1]$.

An analysis of a system with such a scaling will surely be inconclusive, since for example the two similar manipulated inputs are scaled by very different values.

The described procedure to obtain a scaling independent representation involves that the variance of the signals during operation of the system must be known. The inputs to the process need to be excited with signals with selected variances. These excitation variances should be selected according to the expected or allowed change in the process inputs. The process outputs are then measured/simulated, and the variances of the output signals are estimated.

In the case of normally distributed variables, the standard deviation gives a $95 \%$ confidence interval for its value. So a good rule when the inputs are excited with white noise is to select the variance of the input excitation as the square of the maximum expected deviation from the average value. In the case of the quadruple tank, the analyzed working point has a $50 \%$ opening as nominal value for both pumps. A variation of $\pm 10 \%$ is considered, which gives a variance of 100 in the case of $u_{2}$, since it is expressed in \%, and a variance of 0.01 in the case of $u_{1}$, since it is scaled by a factor of 100 .

Since the scaled process disturbances are allowed to vary within the interval $[-1,1]$ around the working point, the excitation of the process disturbances is selected to be white noise with a variance of $(1 / 3)^{2}$, which means that $99.7 \%$ of the randomly generated values will be within the allowed interval, and outliers have been saturated.

The variance of the scaled input signals (manipulated inputs and process disturbances) is therefore

$\boldsymbol{\sigma}_{\bar{u}}^{2}=\operatorname{diag}\left(0.01,100,\left(\frac{1}{3}\right)^{2},\left(\frac{1}{3}\right)^{2}\right)$

The output signals were measured after the scaling in Eq. (5b) was applied, and the variance of the signals was collected in:

$\sigma_{\bar{y}}^{2}=\operatorname{diag}(6.5780 e-004,0.0298,0.0036,0.0011)$

The scaling independent representation can therefore be created as $\tilde{\boldsymbol{Q}}=\left(\boldsymbol{\sigma}_{\bar{y}}^{-1} D_{y}^{-1} \boldsymbol{\Phi} D_{y} \boldsymbol{\sigma}_{\bar{y}}, \boldsymbol{\sigma}_{\bar{y}}^{-1} D_{y}^{-1} \boldsymbol{\Gamma} D_{u} \boldsymbol{\sigma}_{\bar{u}}\right)$, with input-output matrix $\tilde{\boldsymbol{\Omega}}=\boldsymbol{\sigma}_{\bar{y}}^{-1} D_{y}^{-1} \boldsymbol{\Omega} D_{u} \boldsymbol{\sigma}_{\bar{u}}$.

The methods for the analysis of complex processes introduced in this paper are applied in the next section to the scaling independent visualization of the quadruple tank. Even if the method for creating the scaling independent visualization has originally been designed for linear processes, the experiment previously described for measuring the variance of the input and output signals was performed on a simulation of the non-linear quadruple tank around the selected working point with satisfactory results.

\section{Norms and normalizations for quantifying the significance of the process interconnections}

Gramian based IMs are Index Arrays (IAs) and are largely used for determining feasible control structures. In these IAs, first a norm-based operator is applied to each of the input-output subsystems in order to quantify the significance of the represented interconnection. Traditionally used operators include the Hankel norm [21,6], the squared Hilbert-Schmidt norm [7], and the $\mathcal{H}_{2}$ norm [18]. The IA is then computed by normalizing the significance of the input-output channels:

$[I A]_{i j}=\frac{\left[G_{i j}(s)\right]_{p}}{\sum_{i, j=1}^{m, n}\left[G_{i j}(s)\right]_{p}}$

where $[\cdot]_{p}$ denotes the selected operator, and $G_{m \times n}(s)$ is the multivariable transfer function matrix. The term $s$ used to represent the dependence on the Laplace variable for continuous time systems will be dropped in the sequel for the sake of simplicity. The notation $G(\mathbf{j} \omega)$ will be used in the sequel to specify the evaluation of the transfer function in the frequency domain.

As a result of the normalization all the elements of any of these IAs add up to one. The selection of the control structure is made by selecting a subset of the most important interconnections, which will form a reduced model on which control will be based.

In the methods created in this paper, the squared $\mathcal{H}_{2}$ norm is used for quantifying the importance of the process interconnections due to the reasons which will be discussed in Section 5.1. When the process is to be analyzed in frequency domain, the squared magnitude of the transfer functions will be used to represent the importance of the process interconnections.

Besides, instead of using the traditional normalization in Eq. (6), row and column normalizations will be used in the analysis as later described in Section 5.2.

\subsection{The use of the $\mathcal{H}_{2}$ norm for quantifying the significance of the process interconnections}

For any of the $G_{i j}(s)$ SISO subsystems of a multivariable process described by a transfer function $G_{m \times n}(s)$, if $G_{i j}(s)$ is stable and strictly proper, $\left\|G_{i j}(s)\right\|_{2}$ can be expressed as

$\left\|G_{i j}\right\|_{2}=\sqrt{\frac{1}{2 \pi} \int_{-\infty}^{\infty}\left|G_{i j}(\mathbf{j} \omega)\right|^{2} d \omega}$

The reasons why the $\mathcal{H}_{2}$ norm was selected over the HilbertSchmidt or the Hankel norms to quantify the significance of the process interconnections are:

- The frequency dependent methods $\operatorname{SDPT}(\omega)$ and $\operatorname{FDPT}(\omega)$ to be here presented, describe the power conversion at the process interconnections for each frequency $\omega$. Using the $\mathcal{H}_{2}$ norm allows the compression of this information into real valued numbers which can be used to create directed graphs, since it is obtained by integrating the power conversion with respect to the frequency as it can be observed in Eq. (7).

- An interpretation is that the squared $\mathcal{H}_{2}$ norm of a SISO system can be interpreted as the coupling in terms of the energy transmission rate between the past inputs and the current output [22]. Therefore SISO elementary subsystems in a multivariable system with 
larger value of the $\mathcal{H}_{2}$ norm identify the input-output channels which are able to exchange larger amounts of energy.

- It has been shown in [7] that the squared Hilbert-Schmidt norm of a system increases with the presence of time-delays. The same fact can also be shown for the Hankel norm (see A). This means that the larger the time delay in an input-output channel, the larger the channel significance quantified by these two operators. This is an inconvenient property for a norm quantifying the channel significance, since previous simulation work in [22] indicate that the presence of a time delay by itself is not a reason enough to say that a particular input-output pair should be included in the controller when a decentralized controller structure is desired. It was therefore decided that the $\mathcal{H}_{2}$ norm is a more convenient measure for the channel significance due to its insensitivity to channel delays.

\subsection{Normalizations used to compare the significance of the process interconnections}

We will further use the row normalization $\Sigma_{\triangleright}(A)$ and the column normalization $\Sigma_{\nabla}(A)$ on a matrix $A \in \mathbb{R}^{m \times n}$ :

$$
\begin{gathered}
{\left[\Sigma_{\triangleright}(A)\right]_{i j} \triangleq\left\{\begin{array}{cc}
0 & , \text { if } \sum_{l=1}^{n} A_{i l}=0 \\
\frac{A_{i j}}{\sum_{l=1}^{n} A_{i l}} & , \text { otherwise }
\end{array}\right.} \\
{\left[\Sigma_{\nabla}(A)\right]_{i j} \triangleq\left\{\begin{array}{cc}
0 & , \text { if } \sum_{k=1}^{m} A_{k j}=0 \\
\frac{A_{i j}}{\sum_{k=1}^{m} A_{k j}} & , \text { otherwise }
\end{array}\right.}
\end{gathered}
$$

Note that these normalizations involve that the rows of $\Sigma_{\triangleright}(A)$ either add up to 1 or all the elements of the row are 0 , whereas the columns of $\Sigma_{\nabla}(A)$ either add up to 1 or all the elements of the column are 0 .

We will denote by $\left[\left|G_{i j}(\mathbf{j} \omega)\right|^{2}\right]$ to the result of collecting in a matrix the squared magnitude of each of the elementary transfer functions of a multivariable system $G_{m \times n}$. The normalizations $\Sigma_{\triangleright}\left(\left[\left|G_{i j}(\mathbf{j} \omega)\right|^{2}\right]\right)$ and $\Sigma_{\nabla}\left(\left[\left|G_{i j}(\mathbf{j} \omega)\right|^{2}\right]\right)$ will be used to obtain a relative measure of the significance of the process interconnections in the frequency domain.

We will denote as $\left[\left\|G_{i j}\right\|_{2}^{2}\right]$ to the real valued matrix result of collecting the squared $\mathcal{H}_{2}$ norm of each of the elementary subsystems of the multivariable system $G_{m \times n}$. The normalizations $\Sigma_{\triangleright}\left(\left[\left\|G_{i j}\right\|_{2}^{2}\right]\right)$ and $\Sigma_{\nabla}\left(\left[\left\|G_{i j}\right\|_{2}^{2}\right]\right)$, and will be used to obtain an overall measure of the relative significance of the process interconnections.

This means that $\Sigma_{\triangleright}\left(\left[\left|G_{i j}(\mathbf{j} \omega)\right|^{2}\right]\right)$ compares the elements of the same row of $\left[\left|G_{i j}(\mathbf{j} \omega)\right|^{2}\right]$ in frequency domain, whilst $\Sigma_{\triangleright}\left(\left[\left\|G_{i j}\right\|_{2}^{2}\right]\right)$ compares the squared $\mathcal{H}_{2}$ norm of the elements in the same row of $G$. In a similar way the comparisons of $\Sigma_{\nabla}\left(\left[\left|G_{i j}(\mathbf{j} \omega)\right|^{2}\right]\right)$ and $\Sigma_{\nabla}\left(\left[\left\|G_{i j}\right\|_{2}^{2}\right]\right)$ are performed through the columns.

\section{Methods for structural and functional analysis formulated from a visualization representation}

In this section, first an explanation of the difference between structural and functional analysis is given in Section 6.1, and then the structural and functional methods introduced in [23] are formulated from a visualization representation of the system in Section 6.2 and Section 6.3 respectively. Finally, in Section 6.4 the advantages of using the new formulation are enumerated.

\begin{tabular}{|c|c|c|c|c|c|c|c|c|}
\hline & $u_{1}$ & $u_{2}$ & $d_{1}$ & $d_{2}$ & $h_{1}$ & $h_{2}$ & $h_{3}$ & $h_{4}$ \\
\hline$h_{1}$ & 1 & 0 & 0 & 0 & 0 & 0 & 1 & 0 \\
\hline$h_{2}$ & 0 & 1 & 0 & 0 & 0 & 0 & 0 & 1 \\
\hline$h_{3}$ & 0 & 1 & 1 & 0 & 0 & 0 & 0 & 0 \\
\hline$h_{4}$ & 1 & 0 & 0 & 1 & 0 & 0 & 0 & 0 \\
\hline
\end{tabular}

Fig. 2. Structural matrix for the quadruple tank process in Fig. 1.

\subsection{Structural properties vs. functional properties}

\subsubsection{Structural}

The word structure in analysis of systems is related to the concept of causality. When a model of each of the process interconnections (one on one interconnections) between the considered inputs, outputs and internal states is described, then the representation of the process is a structural realization. That is, a structural realization of the quadruple tanks process in Fig. 1 would be the one described in equation (3), where each of the internal one on one interconnections represented in the figure by a red edge is modeled as a transfer function.

When a matrix relating process variables is filled with boolean elements representing whether there is a causal dependence of the variables or not, it is named structural matrix [24]. The structural matrix for the quadruple tank process is represented in Fig. 2.

Structural matrices have been used in [24] as a tool to determine feasible control structures based on the concept of controllability and observability. Visualization of the cause/effect relationship of the process was also provided by associating structural matrices to digraphs. Further work on structural matrices and digraphs is discussed in [25] and [26].

Structural matrices are used as a first stage tool to extract information of the plant which helps to take decision on the control structure. In Section 6.2 we aim to substitute the boolean types in the structural matrices by either a frequency domain description of the relative effect between variables or a real valued weight describing this effect in order to associate the structural matrix with a weighted digraph like the one depicted in Fig. 3.

\subsubsection{Functional}

The word functional is relating to function as opposed to structure. A structural realization of a process would collect all the one on one internal connections of the process, whilst a functional

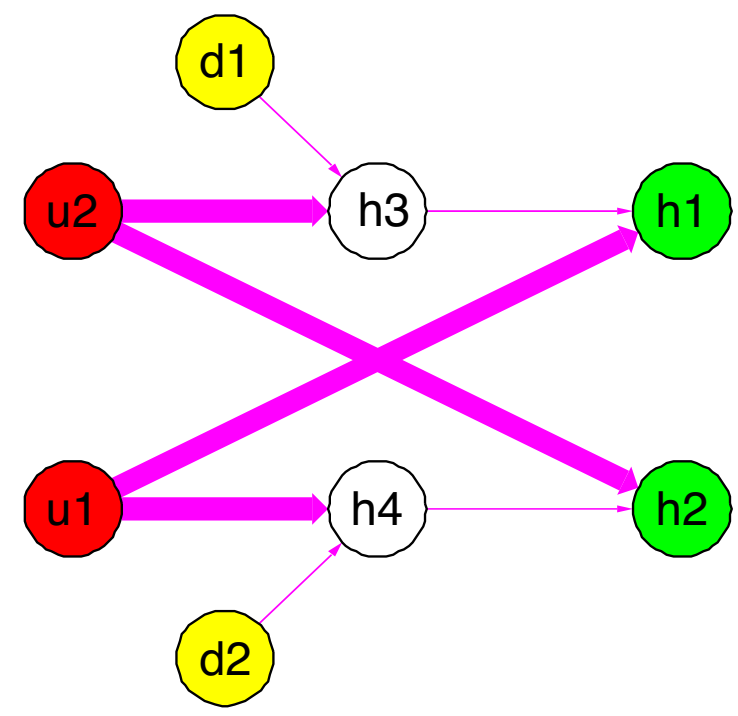

Fig. 3. Structural graph describing the quadruple tank process. The graph was generated with the method SET. ProMoVis screenshot. 


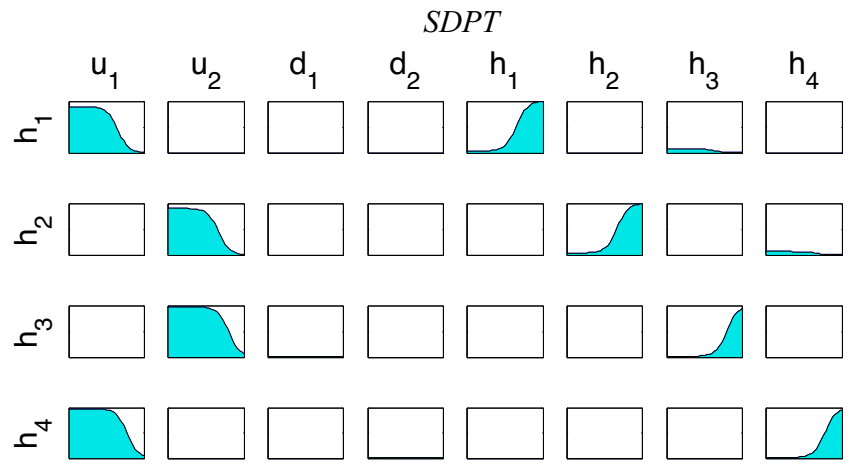

Fig. 4. SDPT for the quadruple tank process for frequencies between $10^{-3} \mathrm{rad} / \mathrm{s}$ and $10^{0} \mathrm{rad} / \mathrm{s}$ (logarithmic scales). The ordinates axes are ranged between 0 and 1 (normalized). ProMoVis screenshot.

realization directly expresses the input-output relationships. A functional realization is therefore representing the total dynamic behavior connecting each of process inputs with each of the process outputs. Each of these input/output connections can be represented by a transfer function which doesn't reflect the process structure, but is instead the combined contribution of all the internal one on one connections including the possible feedback paths. A functional realization of the quadruple tank would be the one in Eq. (4). Analyzing such a realization would be performing a functional analysis of the process. An example of such an analysis can be observed in Fig. 5(right), where the functional effect of independent variations on the process inputs is analyzed.

\subsection{Structural methods}

\subsubsection{Structural energy transfer (SET)}

SET was introduced in [23] as a normalization on a matrix which collects the squared $\mathcal{H}_{2}$ norm of each of the transfer functions representing all the structural interconnections of the process. From a visualization of a system $\boldsymbol{Q}=(\boldsymbol{\Phi}, \boldsymbol{\Gamma})$, in which all the self-references have been hidden, SET can be expressed as:

$S E T \triangleq \Sigma_{\triangleright}\left(\left[||[\boldsymbol{\Phi} \mid \boldsymbol{\Gamma}]_{i j} \|_{2}^{2}\right]\right)$

The element $S E T_{i j}$ describes the direct coupling (in terms of energy transmission rate) between the past values of the variable represented in column $j$ into the future values of $x_{i}$ compared to the coupling of the rest of the variables which are directly affecting $x_{i}$. SET is a real valued matrix, and can be associated with a weighted directed graph which expresses the process causal relationships.
Example 2. Consider the visualization of the quadruple tank described in Eq. (3). Applying SET to its scaling invariant representation yields

SET $=\left(\begin{array}{cccccccc|c}u_{1} & u_{2} & d_{2} & d_{2} & h_{1} & h_{2} & h_{3} & h_{4} & \\ \hline 0.9244 & 0 & 0 & 0 & 0 & 0 & 0.0756 & 0 & h_{1} \\ 0 & 0.9342 & 0 & 0 & 0 & 0 & 0 & 0.0658 \\ 0 & 0.9898 & 0.0102 & 0 & 0 & 0 & 0 & 0 & h_{2} \\ 0.9902 & 0 & 0 & 0.0098 & 0 & 0 & 0 & 0\end{array}\right) \mid \begin{aligned} & h_{3} \\ & h_{4}\end{aligned}$

which determines the weights of the directed graph in Fig. 3. Note that the operation performed is a normalization from the point of view of the sinks of the effect, implying that the weight of all the edges entering a node add up to 1 .

SET and its associated graph provide a structural description of the process, allowing to visualize qualitative and quantitative information about the cause/effect relationship in the plant. Inspecting the graph in Fig. 3 it can be concluded that:

- Pump 1 is directly affecting only to the level of Tanks 1 and 4.

- Pump 2 is directly affecting only to the level of Tanks 2 and 3.

- The variation of level in Tank 3 is only affecting to the variation of level in Tank 1.

- The variation of level in Tank 4 is only affecting to the variation of level in Tank 2.

- Process disturbance $d_{1}$ is directly affecting only to the level of Tank 3, although this effect is insignificant in comparison with the direct effect from Pump 2.

- Process disturbance $d_{2}$ is directly affecting only to the level of Tank 4, although this effect is insignificant in comparison with the direct effect from Pump 1.

\subsubsection{Structural dynamic power transfer (SDPT)}

When a frequency domain description of the cause/effect relationships represented by SET is required, then the method SDPT can be applied. Having a visualization of a system $\boldsymbol{Q}=(\boldsymbol{\Phi}, \boldsymbol{\Gamma})$, SDPT can be expressed as:

$\operatorname{SDPT}(\omega) \triangleq \Sigma_{\triangleright}\left(\left[\left|[(I-\boldsymbol{\Phi}(\mathbf{j} \omega)) \mid \boldsymbol{\Gamma}(\mathbf{j} \omega)]_{i j}\right|^{2}\right]\right)$

The element $S D P T_{i j}$ represents the influence of the past values of the variable represented in column $\mathrm{j}$ into the future values of $x_{i}$ compared to the influence of the rest of the variables which are directly affecting $x_{i}$. The element SDPT $T_{i(i+n)}$, which is relating $x_{i}$ with itself represents the own dynamics of the variable $x_{i}$, that is, how the past values of $x_{i}$ couple to the future ones compared to the coupling from the other variables which are directly affecting to $x_{i}$.
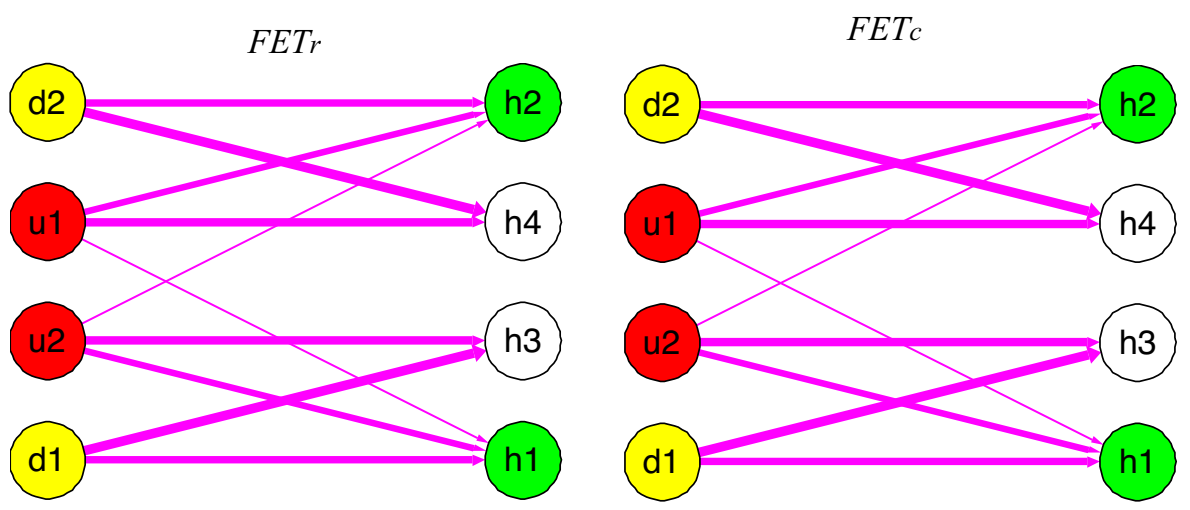

Fig. 5. Graphs associated with $F E T_{r}$ (left) and $F E T_{c}$ (right) for the quadruple tank process. ProMoVis screenshot. 
Example 3. Applying SDPT to the quadruple tank process results in Fig. 4.

SET in Eq. (8) and its associated graph in Fig. 3 provide a structural description of the process, allowing to visualize qualitative and quantitative information about the cause/effect relationship in the plant. When information is required in frequency domain, the matrix SDPT in Fig. 4 can be inspected. The conclusions drawn in the previous example can also be obtained from Fig. 4 . The disadvantage of SDPT over SET is that a graph representation is more intuitive, and the advantage is that the effects are compared in the frequency domain, and therefore additional conclusions can be extracted. An additional conclusion is for example that at high frequencies it becomes hard to influence the level of the tanks. This fact is in concordance with the concept of system bandwidth.

Hence by applying SET and FDPT the structure of the plant is described and direct effects compared, giving a first intuitive analysis of the process.

\subsection{Functional methods}

The previous methods are based on the direct causality of the plant, however, they give limited information about the following questions:

- how is the effect in one of the inputs propagated through the rest of the process?

- how is an internal or controlled variable affected by the process inputs?

The functional methods described in [23] can be used to obtain a numerical analysis and visual representation of these effects.

\subsubsection{Functional energy transfer (FET)}

The name FET was introduced in [23] to designate two different methods for the analysis of functional relationships between process inputs and outputs. Both methods are normalizations on a matrix which collects the squared $\mathcal{H}_{2}$ norm of each of the transfer functions representing all the input-output functional relationships. From a visualization of a system $\boldsymbol{Q}=(\boldsymbol{\Phi}, \boldsymbol{\Gamma})$, with input-output matrix $\boldsymbol{\Omega}$ the methods $F E T_{r}$ and $F E T_{c}$ can be formulated as:

$$
F E T_{r} \triangleq \Sigma_{\triangleright}\left(\left[\left\|\boldsymbol{\Omega}_{i j}\right\|_{2}^{2}\right]\right) ; \quad F E T_{c} \triangleq \Sigma_{\nabla}\left(\left[\left\|\boldsymbol{\Omega}_{i j}\right\|_{2}^{2}\right]\right)
$$

Each of the columns of $F E T_{c}$ is associated with a process input, which in the case of an open loop process, can be a manipulated variable or a process disturbance. Each of the columns of $F E T_{c}$ associated with a manipulated variable represents the relative effect of the manipulated variable onto the rest of the process, each of the columns associated with a process disturbance represents how the process disturbance is propagated through the rest of the process.

Each of the rows of $F E T_{r}$ is associated to an internal or output variable, and represents the relative effect of the process inputs (manipulated variables and process disturbances in open loop case) onto the corresponding internal or output variable.

In both cases, the effect is quantified in terms of energy transfer rate by using the squared $\mathcal{H}_{2}$ norm.

Example 4. The methods SET and SDPT were used in the previous section to describe the structure of the plant and compare the direct effects. Nevertheless, it can be noticed for example that Pump 1 also affects the level of Tank 2, or that the process disturbance $d_{2}$ also affects to the level of Tank 2. The questions at the starting of Section 6.3 then arise.
Applying $F E T_{C}$ to the quadruple tank results in

$F E T_{c}=\left(\begin{array}{cccc|c}u_{1} & u_{2} & d_{2} & d & \\ \hline 0.1180 & 0.3854 & 0.4364 & 0 \\ 0.3854 & 0.1167 & 0 & 0.4369 \\ 0 & 0.4979 & 0.5636 & 0 & h_{1} \\ h_{2} \\ h_{3} \\ h_{4}\end{array}\right.$

which can be translated in the weighted graph in Fig. 5 (right). Each of the columns describes how the energy at the process inputs is propagated through the rest of the process. It can then be concluded that:

- Manipulating $u_{1}$ creates an impact in $h_{1}, h_{2}$ and $h_{4}$. The impact on $h_{1}$ being very low compared with the two other.

- Manipulating $u_{2}$ creates an impact in $h_{1}, h_{2}$ and $h_{3}$. The impact on $h_{2}$ being very low compared with the two other.

- A variation in $d_{1}$ creates similar impacts in $h_{1}$ and $h_{3}$. The impact on $h_{3}$ being slightly more significant.

- A variation in $d_{2}$ creates similar impacts in $h_{2}$ and $h_{4}$. The impact on $h_{4}$ being slightly more significant.

Applying $F E T_{r}$ to the quadruple tank results in

$F E T_{r}=\left(\begin{array}{cccc|c}u_{1} & u_{2} & d_{2} & d & \\ \hline 0.2222 & 0.7699 & 0.0079 & 0 & h_{1} \\ 0.7512 & 0.2413 & 0 & 0.0074 & h_{2} \\ 0 & 0.9898 & 0.0102 & 0 & h_{3} \\ 0.9902 & 0 & 0 & 0.0098 & h_{4}\end{array}\right.$

which can be translated in the weighted graph in Fig. 5 (left). Each of the rows of FETr describes the relative effect into an internal or output variable from the process inputs. It can then be concluded that:

- $h_{1}$ is mainly affected by the pump action $u_{2}$ with a slight effect from $u_{1}$. The effect from the process disturbance $d_{1}$ is very low and can be neglected in comparison with the one from $u_{2}$.

- $h_{2}$ is mainly affected by the pump action $u_{1}$ with a slight effect from $u_{2}$. The effect from the process disturbance $d_{2}$ is very low and can be neglected in comparison with the one from $u_{1}$.

- $h_{3}$ is affected by the pump action $u_{2}$, and by the process disturbance $d_{1}$. The effect of $d_{1}$ is insignificant compared with the effect from $u_{2}$

- $h_{4}$ is affected by the pump action $u_{1}$, and by the process disturbance $d_{2}$. The effect of $d_{2}$ is insignificant compared with the effect from $u_{1}$

\subsubsection{Functional dynamic power transfer (FDPT)}

When a frequency domain description of these effects is required, the method Functional Dynamic Power Transfer (FDPT) can be used.

$$
\operatorname{FDPT}_{r}(\omega)=\Sigma_{\triangleright}\left(\left[\left|\boldsymbol{\Omega}_{i j}(\mathbf{j} \omega)\right|^{2}\right]\right) ; \quad \operatorname{FDPT}_{c}(\omega)=\Sigma_{\nabla}\left(\left[\left|\boldsymbol{\Omega}_{i j}(\mathbf{j} \omega)\right|^{2}\right]\right)
$$

Example 5. The result of applying FDPT to the quadruple tank is depicted in Fig. 6.

Deeper insight is then obtained in the functional analysis of the quadruple tank process. It can be observed in the first row of FDPTr that, as noticed before, the main effect on $h_{1}$ comes from $u_{2}$, nevertheless, this effect is dominating at low frequencies. At high frequencies (above the cross-over frequency) there is a higher contribution on $h_{1}$ from $u_{1}$ than from $u_{2}$. In a similar way, for $h_{2}$ it is $u_{1}$ dominating at low frequencies and $u_{2}$ at high frequencies. 


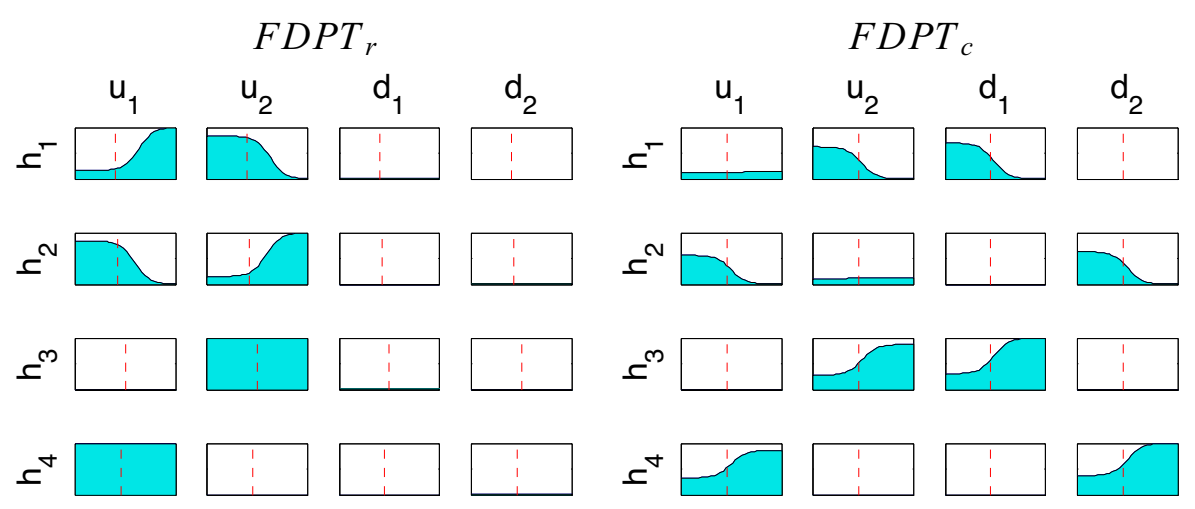

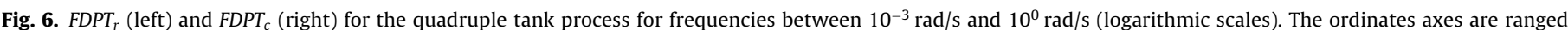

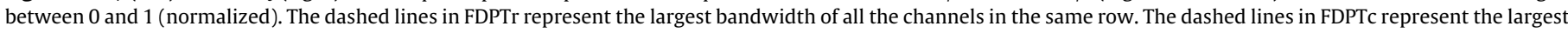
bandwidth of all the channels in the same column. ProMoVis screenshots.

\subsection{Advantages of the new formulation}

- Simpler and more general. The new formulation uses a much more general representation of the process. The previous formulation required a representation of the process in an obscure realization which is sometimes hard to formulate.

- Based on signal flow graphs theory. This allowed a simpler and more versatile implementation in the software tool ProMoVis [15], where the process models are defined by the user through a visual interface which allows to introduce dynamic models (edges) connecting the process variables (nodes) like in Fig. 1. When the structural or functional methods are applied, the result can be translated into weighted graphs like the ones in Fig. 3 or Fig. 5.

- Operations are defined for hiding nodes. This allows hiding the nodes representing variables which are not of interest in the analysis.

- Process disturbances can be driven through filters. It is assumed that the process disturbances in this representation are white noise. Nevertheless, it is easy to describe colored noise, for example, by creating an internal variable which is defined as the white noise driven through a filter describing the frequency content of the colored noise. This internal variable (node) can afterwards be hidden, since it will not be a relevant variable in the analysis.

- Analysis at a selected range of frequencies for the actuators excitation. Sometimes it is of interest to obtain graphs which describe the relative contribution of the manipulated inputs at a certain range of frequencies, for example, at frequencies around the crossover frequency, since it is the frequencies at which control is active. This can easily be done (like in the case of filtering the process disturbances) by pre-multiplying the process inputs by a filter which passes the frequencies of interest and then applying the described methods to the resulting representation of the process.

- Allows open loop and closed loop analysis. The high versatility of the formulation allows easy computation of open loop analysis, but also of closed loop analysis as it is described in Section 9. This is a clear improvement, since the original methods were only described for open loop analysis.

- The use of the operator $z$ instead of $s$ can be used to represent discrete time systems with a given sample time. This is a clear improvement from the initial formulation, since the methods where originally introduced only for continuous time systems.

\section{Interpretation of the methods and applications in open loop analysis}

When a linear model of the process is available, the structural analysis of the plant described in Section 6.2 gives a qualitative and quantitative description of the cause/effect relationships of the process around the working point.

If the propagation of the effects from the manipulated variables and process disturbances is to be analyzed, the methods in Section 6.3 are applied. The columns of $F D P T_{C}$ and $F E T_{C}$ show the distribution of the effects of a manipulated input or process disturbance in the process. The rows of FDPT $r$ and $F E T_{r}$ compare the effects on a certain internal or output variable from the manipulated inputs and process disturbances.

\subsection{Applications in control structure selection}

The method $F E T_{r}$ combined with its counterpart in the frequency domain $F D P T_{r}$, have been previously used to successfully design control structures by considering only the input-output connections which were found to be significant $[23,15]$.

Using $F E T_{r}$, a controlled variable should be associated with the minimum number of actuators that result in a large enough value of the sum of their contributions (edge widths). In general, there is no theory stating which is the minimum sum of contributions which will define a control structure able to derive in an acceptable performance. However, based on the experience from [15] and with similar interaction measures [7], we will consider that a value larger than 0.7 should be achieved to be on the safe side. Besides, it is of interest that the structural matrix obtained by considering only the channels between the manipulated inputs and the controlled variables found to be significant, represents a structurally controllable system [27].

Process disturbances can be neglected as long as their contribution is minor in relation to the contribution of the manipulated variables, which is a well-known fact. Thus, control structure selection should combine interconnections to achieve this requirement. If process disturbances are measurable then a large contribution could be circumvented by the incorporation of a feed-forward action. Clearly, the achievable performance is limited when large contributions are neglected. In this framework, the presence of process disturbances can be considered as additional input variables which are contributing to the dynamics of each of the process output variables. When using a row of $F E T_{r}$ to select which input variables will be associated to a controlled variable, only the columns corresponding to the manipulated variables can be selected. The columns related to process disturbances will be considered as a contribution on the dynamics of the controlled variable which is not possible to use in the control structure. Therefore, the rules for the selection of the control structure remain valid, however, it might be possible that more actuator-sensor channels have to be considered in a perturbed process in order to be able to 
generate a combined contribution which is sufficient for satisfactory rejecting the process disturbances whilst presenting low loop interaction.

This approach can be combined with the method FDPT to analyze the effect on the controlled variables in the frequency domain. The decision on the control structure selection can then be validated at the frequency rage in which control is active.

Example 6. A decentralized control structure will be designed for the quadruple tank process. The objective is therefore to pair each of the controlled variables $\left(h_{1}\right.$ and $\left.h_{2}\right)$ with an actuator $\left(u_{1}\right.$ and $\left.u_{2}\right)$. It is clear from the graph obtained with $F E T_{r}$ in Fig. 5 (left), that the preferred decentralized pairing is $u_{1}-h_{2}, u_{2}-h_{1}$. This structure is likely to derive in a good rejection of process disturbances, since it has previously been revealed, that the effect of the process disturbances in the controlled variables is insignificant compared with that of the process inputs. A numerical quantification of these effects can be observed in Eq. (9), where it is confirmed that the channels $u_{1}-h_{2}$ and $u_{2}-h_{1}$ are weighted with values larger than 0.7. Inspecting $F D P T_{r}$ in Fig. 6 (left) it can be observed for the decentralized pairing selected that, at low frequencies, there will be a low amount of interaction between the control loops. This interaction effect starts to increase at frequencies around the crossover frequency, where a large control effort is expected. The fact that the dominating decentralized pairing switches at high frequencies is not relevant, since these frequencies are larger than the crossover frequency.

The Relative Gain Array (RGA), which is probably the most widely used IM will now be used as a comparison with the methodology here proposed. The RGA was introduced in [2], and for a multivariable process with matrix transfer function $G$, it is defined as $R G A(G)=G(0) \otimes G(0)^{-T}$, where $\otimes$ represents element by element multiplication, and $G(0)^{-T}$ is the transpose of the inverse of the DC-gain matrix $G(0)$. The RGA can only be used for the design of decentralized control structures, being the preferred parings those with RGA values close to 1 . Negative values of the RGA should not be selected for pairing due to potential instability issues. The RGA for the subsystem formed by the inputs $u_{1}, u_{2}$ and the outputs $y_{1}, y_{2}$ in is:

$R G A=\frac{u_{1}}{\left(\begin{array}{cc}-0.225 & u_{2} \\ 1.225 & -0.225\end{array}\right)} \mid \begin{aligned} & y_{1} \\ & y_{2}\end{aligned}$

This suggests the same decentralized pairing than the one selected using $F E T_{r}$. By evaluating the value of the RGA in the frequency domain, the decisions on the decentralized controller can be evaluated at any desired range of frequencies. This generalization of the RGA in frequency domain is known as Dynamic RGA (DRGA) [28], and was used in [20] to determine a decentralized control structure for the quadruple tank system. The magnitude of the DRGA for the quadruple tank system is depicted in Fig. 7. In this example, similar conclusions can be obtained from the FDPT $r$ and the DRGA: at low frequencies there exists a moderated amount of interaction between the control loops since the diagonal elements of the DRGA are at the order of 0.225 , the interaction starts to increase around the crossover frequency, and at high frequencies the preferred decentralized pairing switches.

The differences of $F E T_{r}$ and $F D P T_{r}$ with the DRGA are:

- The RGA can only be used for designing decentralized control structures. FET $r$ and $F D P T_{r}$ can be used to design sparse control structures.

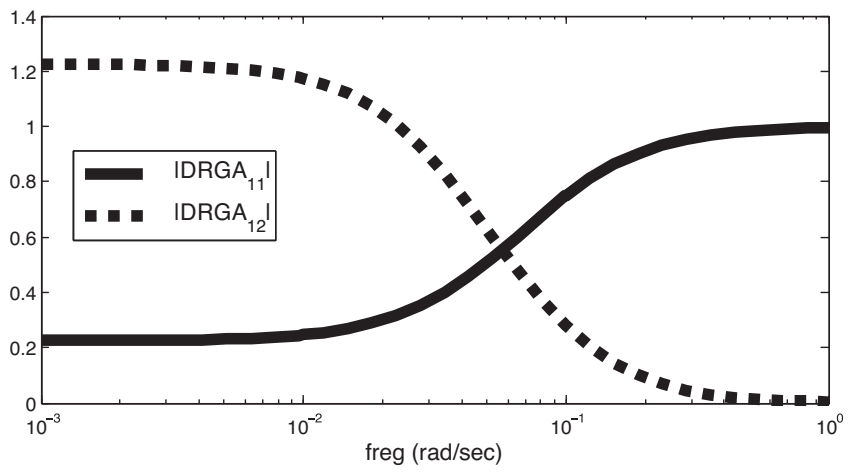

Fig. 7. DRGA for the quadruple tank process.

- The DRGA can only be evaluated as a function of the frequency. $F E T_{r}$ compresses the information in the frequency domain into a real number by the use of the $\mathcal{H}_{2}$ norm.

- The RGA in combination with the Niederlinski Index [29] can be used to assess the feasibility of designing a stable decentralized controller with a certain structure. $F E T_{r}$ and $F D P T_{r}$ only give information about the energy transfer in the process interconnections, no conclusions about the stability of the closed loop system can be derived.

- The RGA is not able to analyze the effect of the process disturbances. $F E T_{r}$ and $F D P T_{r}$ can quantify the importance of the impact of the process disturbances and have the potential to be able to identify cases in which additional interconnections in the controller structure will significantly increase the performance on disturbance rejection.

Example 7. Assume that, in the previous example, the allowed variation of the process disturbance $d_{1}$ is multiplied by a factor of 6. The row in $F E T_{r}$ associated to $h_{1}$ is now:

$$
\left[F E T_{r}\right]_{h_{1}}=\begin{array}{cccc}
u_{1} & u_{2} & d_{1} & d_{2} \\
& \left(\begin{array}{llll}
0.1742 & 0.6036 & 0.2222 & 0
\end{array}\right) & h_{1}
\end{array}
$$

The decentralized control structure selected in the previous example would only reflect around $60 \%$ of the dynamic contribution to the controlled variable $h_{1}$. It is now unlikely to achieve a good performance with the selected structure while keeping the control action $u_{2}$ within an expected variation of $\pm 10 \%$. It is therefore advised to consider the input-output channel $u_{1}-h_{1}$ in the control structure to obtain a dynamic contribution of around $78 \%$ on the controlled variable $h_{1}$. A second option is to increase the expected variation on the control action $u_{2}$ to $\pm 15 \%$, this will be reflected in using a variance for $u_{2}$ of $15^{2}$ in Eq. (5a), and the resulting value of the row in $F E T_{r}$ associated to $h_{1}$ is now:

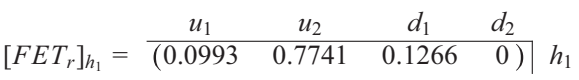

And therefore if the allowed expected variation of $u_{2}$ is increased to $\pm 15 \%$ the original decentralized control structure considers around $77 \%$ of the dynamic contribution on $h_{1}$ and the achievable performance is very likely to be satisfactory.

\section{Scaling sensitivity}

An approach for obtaining a scaling independent representation of a system has been introduced in Section 3. This approach has been applied to create a scaling independent representation of the quadruple tank system in Section 4. The introduced methods for the structural and functional analysis of complex processes have 


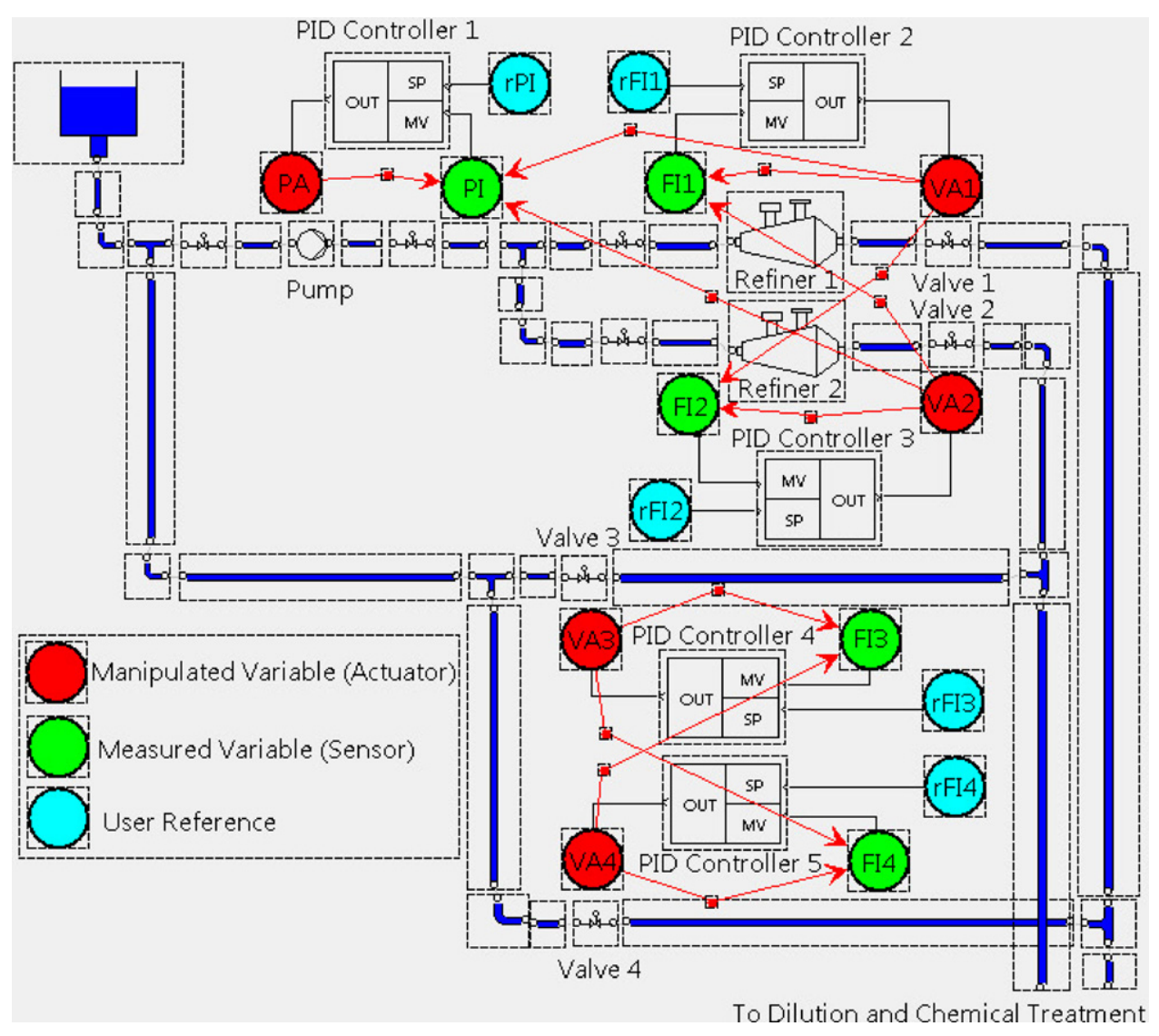

Fig. 8. Refining section of the stock preparation plant at SCA Obbola AB, Sweden. ProMoVis screenshot, Source: [15].

successfully been applied to the scaling invariant representation of the quadruple tank system in Section 6.

However, it is of interest to study the scaling sensitivity of the proposed methods in order to consider the cases in which other traditional scaling techniques are used.

Lemma 2. Let $D_{x}$ and $D_{u}$ be the diagonal scaling matrices of a linear time-invariant MIMO system $\Omega^{m \times n}$. Let T be an operator on a SISO system subsystem $\Omega_{i j}$ such that $T\left(K \cdot \Omega_{i j}\right)=K \cdot T\left(\Omega_{i j}\right)$, with $K \in \mathcal{R}$. Denoting $\left[T\left(\Omega_{i j}\right)^{2}\right]$ the matrix resulting of squaring $T\left(\Omega_{i j}\right)$ for each of the SISO subsystems of $\Omega$, then $\Sigma_{\triangleright}\left(\left[T\left(\Omega_{i j}\right)^{2}\right]\right)$ is invariant to output scaling of $\Omega$, and the operation $\Sigma_{\nabla}\left(\left[T\left(\Omega_{i j}\right)^{2}\right]\right)$ is invariant to input scaling of $\Omega$.

Proof. Both statements are proved with the following identities: of the analysis of the refining section of the stock preparation plant in SCA Obbola AB, Sweden previously published in [15].

In conventional refining, the pulp is pumped through the gap between two grooved discs. A moving disc can be rotated and displaced in the axial direction, and the friction of the fibers with the discs and with each other creates the refining effects. Refining creates major changes in pulp properties. External fibrillation is the most desired of the effects, improving the fibre bonds at the forming section. Refining also creates undesirable effects on the pulp, i.e. internal fibrillation, which has a large impact in the dewatering capacity of the paper web, the shortening of the fibre flocs, which may have a negative impact in the paper forming, and

$$
\begin{aligned}
& \Sigma_{\triangleright}\left(\left[T\left(\left[D_{x}^{-1} \Omega D_{u}\right]_{i j}\right)^{2}\right]\right)=\frac{T\left(\left[D_{x}^{-1} \Omega D_{u}\right]_{i j}\right)^{2}}{\sum_{l=1}^{n} T\left(\left[D_{x}^{-1} \Omega D_{u}\right]_{i l}\right)^{2}}=\frac{\left[D_{x}\right]_{i i}^{-2} T\left(\Omega_{i j}\right)^{2}\left[D_{u}\right]_{j j}^{2}}{\sum_{l=1}^{n}\left[D_{x}\right]_{i i}^{-2} T\left(\Omega_{i l}\right)^{2}\left[D_{u}\right]_{l l}^{2}}=\frac{T\left(\Omega_{i j}\right)^{2}\left[D_{u}\right]_{j j}^{2}}{\sum_{l=1}^{n} T\left(\Omega_{k j}\right)^{2}\left[D_{u}\right]_{l l}^{2}}=\Sigma_{\triangleright}\left(\left[T\left(\left[\Omega D_{u}\right]_{i j}\right)^{2}\right]\right) \\
& \Sigma_{\nabla}\left(\left[T\left(\left[D_{x}^{-1} \Omega D_{u}\right]_{i j}\right)^{2}\right]\right)=\frac{T\left(\left[D_{x}^{-1} \Omega D_{u}\right]_{i j}\right)^{2}}{\sum_{k=1}^{m} T\left(\left[D_{x}^{-1} \Omega D_{u}\right]_{k j}\right)^{2}}=\frac{\left[D_{x}\right]_{i i}^{-2} T\left(\Omega_{i j}\right)^{2}\left[D_{u}\right]_{j j}^{2}}{\sum_{k=1}^{m}\left[D_{x}\right]_{k k}^{-2} T\left(\Omega_{k j}\right)^{2}\left[D_{u}\right]_{j j}^{2}}=\frac{\left[D_{x}\right]_{i i}^{-2} T\left(\Omega_{i j}\right)^{2}}{\sum_{k=1}^{m}\left[D_{x}\right]_{k k}^{-2} T\left(\Omega_{k j}\right)^{2}}=\Sigma_{\nabla}\left(\left[T\left(\left[D_{x}^{-1} \Omega\right]_{i j}\right)^{2}\right]\right)
\end{aligned}
$$

Corollary 1. Note that both operators on linear systems $\|\cdot\|_{2}$ and $\mid$ - | have the same property described for $T(\cdot)$. Therefore, from Eq. (13) and Eq. (14) it can be concluded that:

- FET $r$ and FDPT $T_{r}$ are insensitive to output scaling (scaling of vector $x$ ). - $F E T_{C}$ and $F D P T_{c}$ are insensitive to input scaling (scaling of vector $u$ ).

Example 8. The stock preparation plant is a subprocess present in a paper mill mainly for the refining treatment of chemical and semichemical pulps. The case study included here is an extension the formation of a large amount of fines, which has to be avoided since the fines have to be retained by the paper web at the wire section.

For the refining section of the stock preparation plant in SCA Obbola $A B$, Sweden, the process and the existing controller are depicted in Fig. 8. First the pulp is pumped from a storage tank and the flow bifurcates towards two parallel refiners. Note that a fraction of the pulp is recirculated again for an additional refining. This recirculation increases the complexity of the process, requiring 
Table 3

Considered sensors and actuators in the refining section.

\begin{tabular}{llll}
\hline Tag & Name & Description & Units \\
\hline & & \multicolumn{1}{c}{ Actuators } & \\
PA & Pump Actuator & Pumps the flow through the refiners & $\%$ \\
VA1 & Valve Actuator 1 & Valve after refiner 1 & $\%$ \\
VA2 & Valve Actuator 2 & Valve after refiner 2 & $\%$ \\
VA3 & Valve Actuator 3 & Valve at the recirculation from refiner 2 & $\%$ \\
VA4 & Valve Actuator 4 & Valve at the recirculation from refiner 1 & $\%$ \\
& & Sensors & $\mathrm{kPa}$ \\
PI & Pressure Indicator & Pressure before the flow bifurcation & $\mathrm{l}$ \\
FI1 & Flow Indicator 1 & Pulp flow though refiner 1 & $\mathrm{l} / \mathrm{s}$ \\
FI2 & Flow Indicator 2 & Pulp flow through refiner 2 & $\mathrm{l} / \mathrm{s}$ \\
FI3 & Flow Indicator 3 & Pulp flow recirculated from refiner 2 & $\mathrm{l} / \mathrm{s}$ \\
FI4 & Flow Indicator 4 & Pulp flow recirculated from refiner 1 & \\
\hline
\end{tabular}

a deep analysis of the process interconnections in order to understand the process and to design a control structure.

The set of considered sensors and actuators is summarized in Table 3.

The refiners have internal controllers to track a setpoint for the energy delivered to the pulp. Safety, quality, and production depend on well maintained setpoints for the considered flows and the pressure at the entrance of the refiners. In the current control of the process, four independent scalar PID controllers are used to maintain the flows at the desired setpoints. The centrifugal pump is then used as actuator in another control loop to keep the pressure before the refiners constant.

In order to collect significant process data for the modeling task, the process was excited during normal operation by perturbing the actuators with additive white nose. In a first modeling step, a model structure was created by identifying which actuators generate an observable impact on which measured variables. The actuator-sensor relationships corresponding to this model structure were modeled as dynamic models using system identification techniques. Each of the obtained actuator-sensor models is represented by an arrow in Fig. 8 .

The resulting graph from applying $F E T_{r}$ to the obtained models is depicted in Fig. 9. An optional threshold of 0.1 on the significance of the edges determines their visibility; whilst simplifying the graphical representation and analysis.

From inspection of Fig. 9, it is clear that pairing each of the measured variables with the actuator connected with the most significant edge derives in the best decentralized control structure. This structure is the one already in use in the process, and it is expected to achieve a satisfactory performance. It is also clear at first sight that both recirculation branches can be assumed to behave as independent scalar subprocesses, and simple PID controllers can be independently designed for them in the presence of low loop interaction. Nevertheless, it is suspected that there is

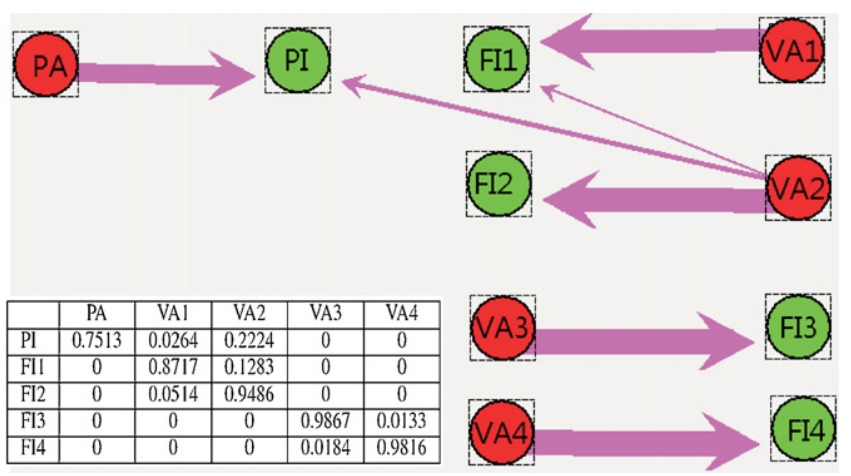

Fig. 9. Connectivity matrix and related graph obtained by applying $F E T_{r}$ to the stock preparation plant. ProMoVis screenshot, Source: [15]. a potential of improving the control performance by considering the dynamic connection from VA2 to PI in the control system, since this will increase the sum of contributions on PI from about 0.72 to 0.97 .

To obtain a deeper insight on the effects on PI, FDPT $r$ is applied, and the result is depicted in Fig. 10. It can be observed that the contribution from VA2 has an important impact at frequencies around the maximum crossover frequency of the considered channels, causing interaction between the control loops which may be translated into oscillations. This conclusion is supported by the fact that the centrifugal pump has rotor dynamics which are slower than the dynamics of the valve, and by the observations of the plant operators and engineers, which confirm the existence of the mentioned oscillations. A potential of improving the existing control structure has therefore been identified. The suggestion is to consider the actuator-sensor connection from VA2 to PI in the control structure, e.g. with a feed-forward action.

The indicators obtained by applying some of the traditional IMs to the stock preparation plant are:

\begin{tabular}{|c|c|c|c|c|c|c|}
\hline & $P A$ & $V A 1$ & $V A 2$ & $V A 3$ & $V A 5$ & \\
\hline \multirow{5}{*}{$R G A=$} & 1 & 0 & 0 & 0 & 0 & $P I$ \\
\hline & 0 & 1.0515 & -0.0515 & 0 & 0 & $F I 1$ \\
\hline & 0 & -0.0515 & 1.0515 & 0 & 0 & $F I 2$ \\
\hline & 0 & 0 & 0 & 1.0170 & -0.0170 & $F I 3$ \\
\hline & 0 & 0 & 0 & -0.0170 & 1.0170 & FI4 \\
\hline \multirow{5}{*}{$P M=$} & 0.7244 & 0.0110 & 0.0288 & 0 & 0 & $P I$ \\
\hline & 0 & 0.0876 & 0.0061 & 0 & 0 & $F I 1$ \\
\hline & 0 & 0.0046 & 0.1337 & 0 & 0 & $F I 2$ \\
\hline & 0 & 0 & 0 & 0.0023 & $1.45 e-005$ & $F I 3$ \\
\hline & 0 & 0 & 0 & $6.7003 e-005$ & 0.0015 & FI4 \\
\hline \multirow{5}{*}{$\Sigma_{2}=$} & 0.4076 & 0.0525 & 0.0851 & 0 & 0 & $P I$ \\
\hline & 0 & 0.1485 & 0.0392 & 0 & 0 & $F I 1$ \\
\hline & 0 & 0.0341 & 0.1835 & 0 & 0 & $F I 2$ \\
\hline & 0 & 0 & 0 & 0.0240 & 0.0019 & $F I 3$ \\
\hline & 0 & 0 & 0 & 0.0041 & 0.0195 & FI4 (15 \\
\hline
\end{tabular}

It can be observed in Eq. (15) that the RGA suggests the same decentralized pairing as the one selected with $F E T_{r}$, however this example illustrates the potential of $F E T_{r}$ and $F D P T_{r}$ for suggesting sparse control structures in interacting systems.

This example also shows the advantage of the $F E T_{r}$ over two gramian based IMs: the PM and the $\Sigma_{2}$. The advantage resides in its insensitivity to output scaling. The inputs to the process are all scaled in \% of actuator effect, which is the usual scaling present in the logged signals of industrial process, and which will be considered to be an adequate scaling. The process outputs are not properly scaled, since besides of being pressure and flow measurements combined, the flows at the recirculation (FI3 and FI4) are only a fraction of the primary flows (FI1 and FI2), and therefore they should be represented at different scales. While $F E T_{r}$ was able to suggest a sparse control structure, the PM and $\Sigma_{2}$ could show inappropriate indications due to its sensitivity to output scaling. From Eq. (15) it can be observed that both the PM and the $\Sigma_{2}$ give a large weight to the channels associated to the variable PI, whilst the channels associated with the recirculation flows FI3 and FI4 receive a rather low weight. This problem is not present in $F E T_{r}$ and $F D P T_{r}$, since only the input-output channels associated with the same output variable are compared, being the sum of all the contributions on an output variable equal to 1 . Despite the inappropriate output scaling, both the PM and the $\Sigma_{2}$ are able to suggest the same decentralized paring than the one previously chosen, being the sum of all the contributions for the decentralized pairing equal to 0.95 for the PM and to 0.64 for the $\Sigma_{2}$. 


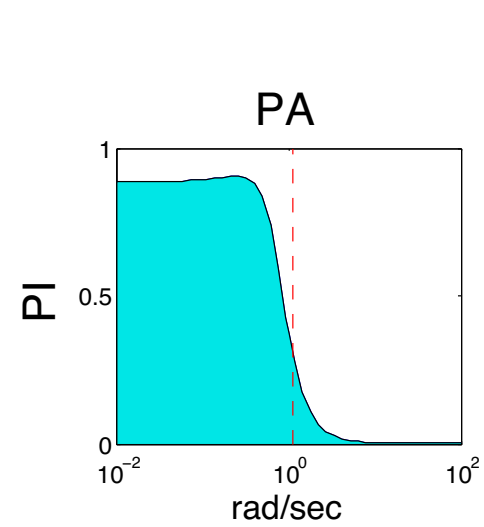

$$
\left[F D P T_{r}\right]_{P I}
$$
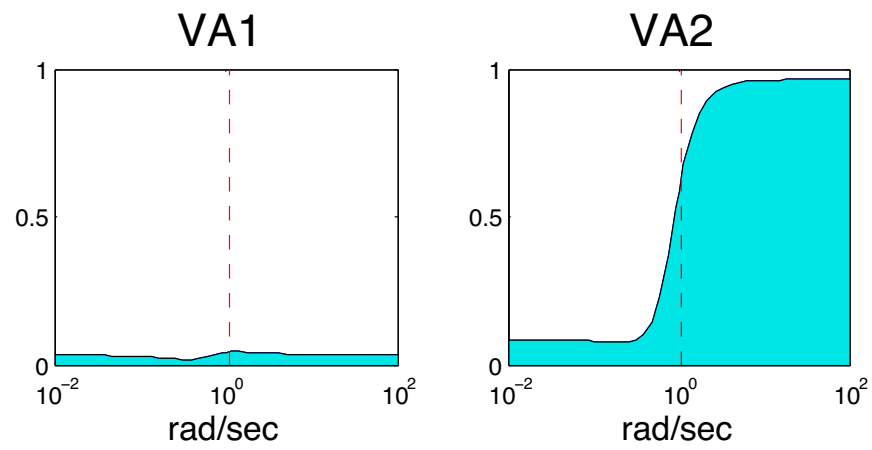

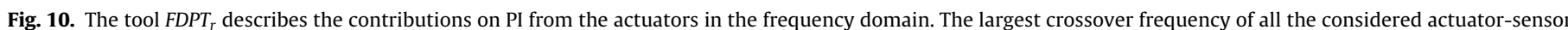
channels is marked by a dashed line. ProMoVis screenshot, Source: [15].

\section{Closed loop analysis}

After control structure design and controller synthesis, the resulting interaction between the control loops can be analyzed by using the functional methods described in this paper. An usual requirement for a multivariable control system is the decoupling of the control loops, which means that when a reference is modified, the effect should be mainly on the measured variable which corresponds to that reference, whilst the effect on the rest of the measured variables corresponding to other control loops should be kept low.

As it will be shown in this section, the introduced functional methods can also help to comprehend the effect of changing the references onto the control actions.

Assume an open loop process represented by a visualization of the form:

$x=\boldsymbol{\Phi} x+\left(\begin{array}{lll}\boldsymbol{\Gamma}_{u} & \mid & \boldsymbol{\Gamma}_{w}\end{array}\right)\left(\begin{array}{c}u \\ w\end{array}\right)$

where the vector $x$ collects measured variables, estimated variables, and internal variables, vector $u$ collects the manipulated variables, and $w$ collects the process disturbances.

Assume that the loop is closed with control action

$u=C_{r} \cdot r-C_{x} \cdot x+C_{F F} \cdot w$

This control action allows a 2-DOF structure with Feed Forward action for the process disturbances.

The closed loop system can then be expressed by a visualization of the form:

$\left(\begin{array}{l}x \\ u\end{array}\right)=\left(\begin{array}{cc}\boldsymbol{\Phi} & \boldsymbol{\Gamma}_{u} \\ -C_{x} & 0\end{array}\right)\left(\begin{array}{l}x \\ u\end{array}\right)+\left(\begin{array}{cc}0 & \boldsymbol{\Gamma}_{w} \\ C_{r} & C_{F F}\end{array}\right)\left(\begin{array}{c}r \\ w\end{array}\right)$

Example 9. Consider an open loop system represented by the following MIMO transfer function:

$G=\left(\begin{array}{cc}\frac{1}{10 s+1} & \frac{0.6}{10 s+1} \\ \frac{-0.6}{s+1} & \frac{1}{10 s+1}\end{array}\right)$

which can be represented by the open loop visualization:

$y=0 \cdot y+G \cdot u$

The system is controlled with a 1-DOF decentralized controller with PI controllers on the diagonal. Both PI controllers have $k_{c}=4$ and $T_{i}=2$.
The closed loop system can now be represented by the visualization, $\boldsymbol{Q}_{C L}=\left(\boldsymbol{\Phi}_{C L}, \boldsymbol{\Gamma}_{C L}\right)$ :

$\underbrace{\left(\begin{array}{l}y \\ u\end{array}\right)}_{z}=\underbrace{\left(\begin{array}{cc}0 & G \\ -C & 0\end{array}\right)}_{\boldsymbol{\Phi}_{C L}} \cdot \underbrace{\left(\begin{array}{l}y \\ u\end{array}\right)}_{z}+\underbrace{\left(\begin{array}{c}0 \\ C\end{array}\right)}_{\boldsymbol{\Gamma}_{C L}} \cdot r ; \quad \boldsymbol{\Omega}_{C L}=\left(I-\boldsymbol{\Phi}_{C L}\right)^{-1} \boldsymbol{\Gamma}_{C L}$

where:

$C=\left(\begin{array}{cc}\frac{k_{c}\left(1+T_{i} \cdot s\right)}{T i \cdot s} & 0 \\ 0 & \frac{k_{c}\left(1+T_{i} \cdot s\right)}{T i \cdot s}\end{array}\right)$

Using the matrix $E_{y}$ for performing a hiding operation on $\boldsymbol{Q}_{C L}$ hides the manipulated variables $u$, and using the matrix $E_{u}$ hides the controlled variables $y$.

$E_{y}=\left(\begin{array}{llll}1 & 0 & 0 & 0 \\ 0 & 1 & 0 & 0\end{array}\right), \quad E_{u}=\left(\begin{array}{cccc}0 & 0 & 1 & 0 \\ 0 & 0 & 0 & 1\end{array}\right)$

To analyze the effect of the references on the controlled variables $(y)$, the functional methods described in 6.3 are applied to the closed loop visualization $\boldsymbol{Q}_{C L}$ after hiding the nodes which correspond to the manipulated variables $(u)$.

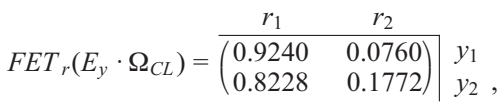

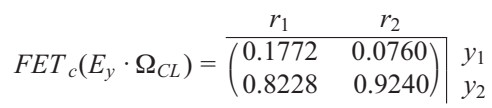

By observing the first column of $F E T_{c}\left(E_{y} \cdot \boldsymbol{\Omega}_{C L}\right)$ in Eq. (16), it can be concluded that a change in $r_{1}$ creates a perturbation on $y_{2}$ which is more significant than the influence on $y_{1} \cdot F D P T_{C}\left(E_{y} \cdot \boldsymbol{\Omega}_{C L}\right)$ in Fig. 11 (right) describes these effects in the frequency domain. By inspecting the first column of $F D P T_{c}\left(E_{y} \cdot \boldsymbol{\Omega}_{C L}\right)$ it can be concluded that the perturbation from the first control loop into $y_{2}$ only exists at high frequencies. Since the interaction does not exist at DC and low frequencies, then a step in any of the references will be tracked at steady state, as it is expected since the decentralized controllers have integral action. Nevertheless, the existing interaction at high frequencies means that a step in the reference $r_{1}$ will largely disturb $y_{2}$ during the transient.

By inspecting the second column of $F E T_{c}\left(E_{y} \cdot \boldsymbol{\Omega}_{C L}\right)$ in Eq. (16), it can be concluded that there is also an impact in $y_{1}$ when the reference of the second control loop is manipulated. Nevertheless, this impact is very low (0.076) compared with the effect on $y_{2}$ $(0.924)$, and it is also existing only at high frequencies as it can 

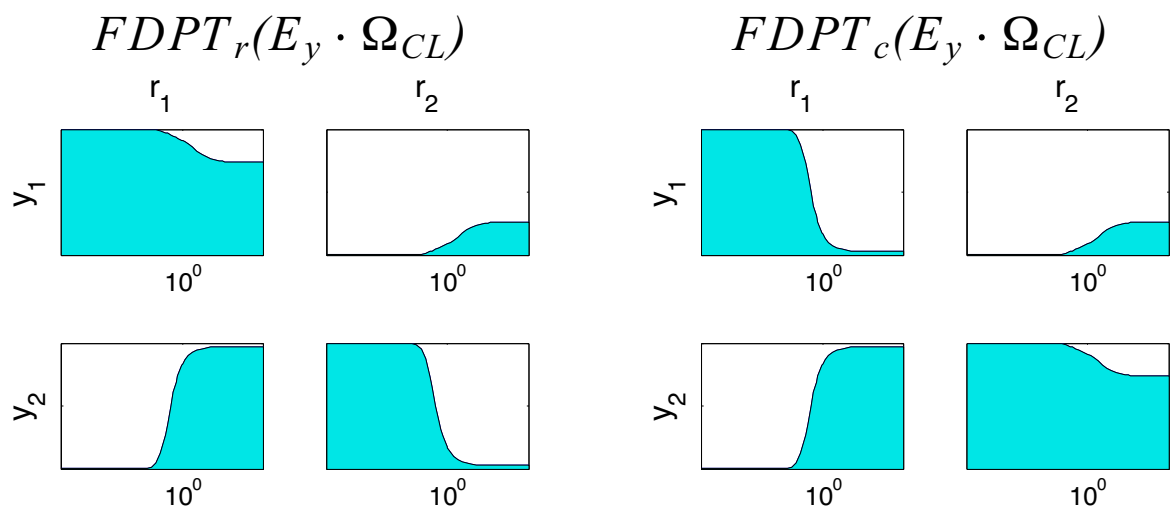

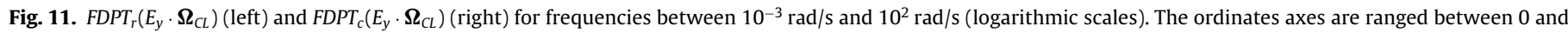
1 (normalized). ProMoVis screenshot.
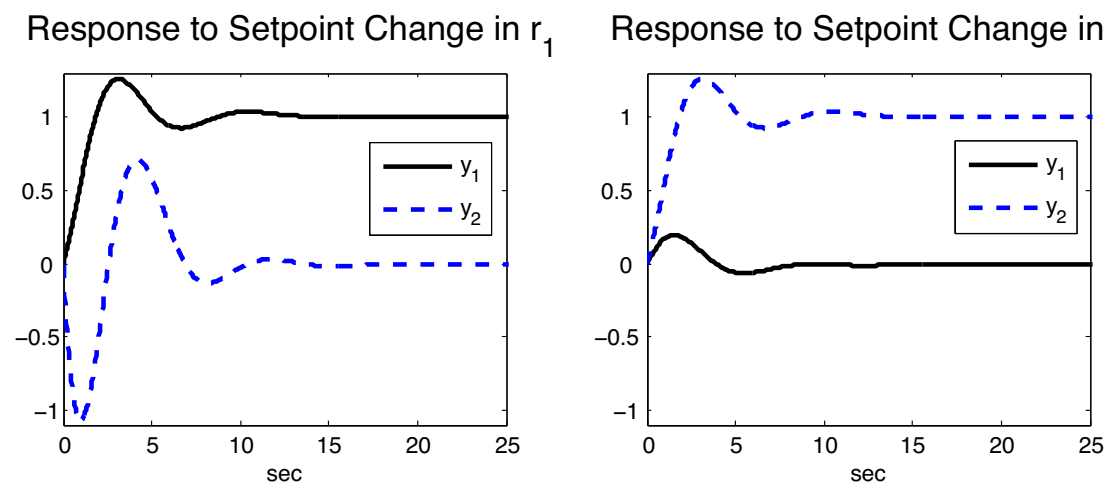

Fig. 12. Responses to setpoint changes for the closed loop system in Example 8.

be appreciated in the second column of $\operatorname{FDPT}_{C}\left(E_{y} \cdot \boldsymbol{\Omega}_{C L}\right)$ in Fig. 11 (right).

The same conclusions were obtained in [30] using loop decomposition, and they were validated by examining the responses to independent setpoint changes in both control loops. These step responses are depicted in Fig. 12.

Furthermore, we will continue the analysis by inspecting $F E T_{r}\left(E_{y} \cdot \boldsymbol{\Omega}_{C L}\right)$ in Eq. (16). Each of the rows of $F E T_{r}$ expresses the relative contribution on an output variable obtained by changing the references. It can be concluded that for equivalent changes in both references, $y_{1}$ is mainly affected by the change in $r_{1}$, being the interaction from manipulating on $r_{2}$ very low. Nevertheless, the second row of $F E T_{r}$ suggests that when both references are subject of equivalent changes, $y_{2}$ receives higher impact from $r_{1}$ than from $r_{2}$. These contributions can be analyzed in frequency domain by inspecting $\operatorname{FDPT}_{r}\left(E_{y} \cdot \boldsymbol{\Omega}_{C L}\right)$, which is depicted in Fig. 11 (left). There it can be observed that even if $y_{2}$ is highly affected by $r_{1}$, this contribution is dominating at high frequencies, whilst at low frequencies and DC it is clear that $y_{2}$ is mainly affected by $r_{2}$.

Therefore, this analysis suggests that, for a simultaneous step change in $r_{1}$ and $r_{2}$, the response of $y_{1}$ will not be much different from that one of only making a step change in $r_{1}$, whilst the response of $y_{2}$ will be highly perturbed compared with the response from only making a step change in $r_{2}$.

These conclusions were validated with the simulations depicted in Fig. 13.

At this point, the effect of setpoint changes on the control actions will be analyzed. Since the resulting closed loop transfer functions from the references to the control actions have the same number of poles and zeros, the $\mathcal{H}_{2}$ norm of these interconnections is not defined. Nevertheless, a filter can be used in order to restrict the
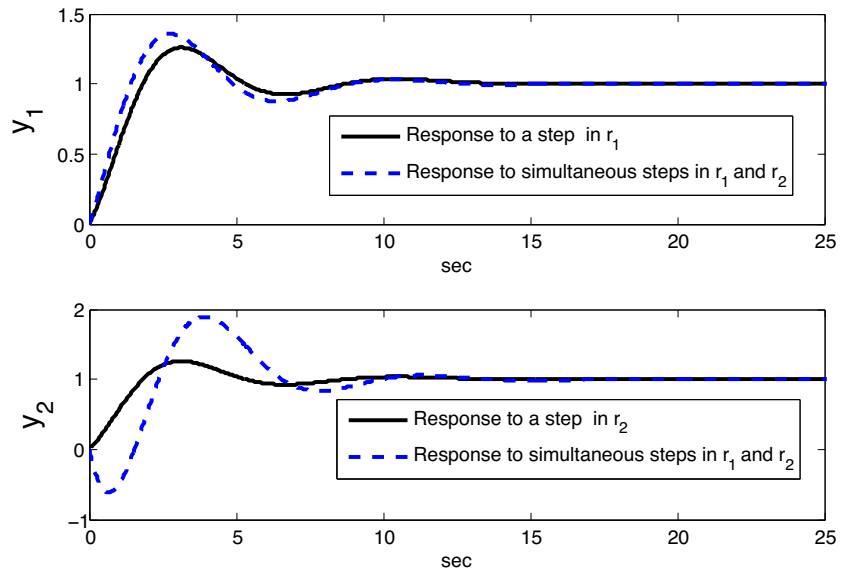

Fig. 13. Analysis of the loop interaction of the closed loop in Example 8 by comparing the response obtained when one reference is changed with that of changing both references simultaneously. ProMoVis screenshot.

analysis to a range of frequencies which are of interest. For this purpose, a second order Butterworth filter with passband between $10^{-1.9} \mathrm{rad} / \mathrm{s}$ and $10^{0.7} \mathrm{rad} / \mathrm{s}$ is used. This frequency range has been selected to include the crossover frequency of the open loop and closed loop processes, since that is the range of frequencies at which the majority of the control action is expected. Denoting the obtained filter as $F, F E T_{c}$ can be computed as:

$F E T_{c}\left(F \cdot E_{u} \cdot \Omega_{C L}\right)=\begin{array}{cc}r_{1} & r_{2} \\ \left(\begin{array}{ll}0.3895 & 0.0323 \\ 0.6105 & 0.9677\end{array}\right) & u_{1} \\ u_{2}\end{array}$ 

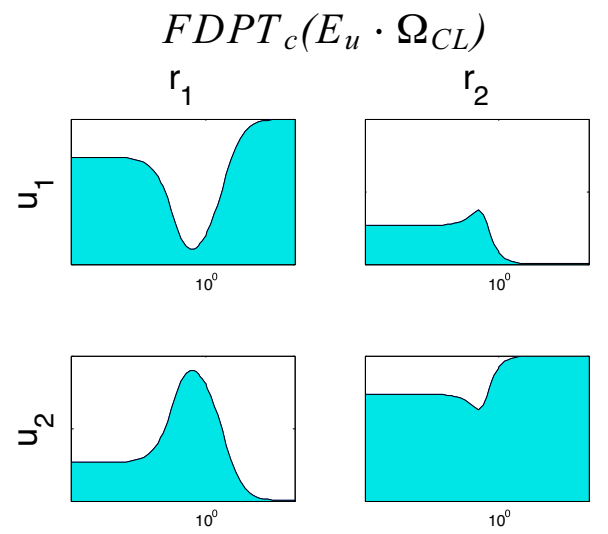

Fig. 14. $F D P T_{c}\left(E_{u} \cdot \boldsymbol{\Omega}_{C L}\right)$ for the closed loop system in Example 8 for frequencies between $10^{-3} \mathrm{rad} / \mathrm{s}$ and $10^{2} \mathrm{rad} / \mathrm{s}$ (logarithmic scales). The ordinates axes are ranged between 0 and 1 (normalized). ProMoVis screenshot.

This indicator suggests that a change either in $r_{1}$ or $r_{2}$ will produce a larger control effort in $u_{2}$ than in $u_{1}$. This effect can be analyzed in frequency domain by inspecting $F D P T_{c}$ in Fig. 14. By inspecting the first column in the figure we can conclude that a setpoint change in $r_{1}$ will require a larger dc variation in the control action of $u_{1}$ than the variation in $u_{2}$, however, there is a peak dominating the effect on $u_{2}$ around the crossover frequency, which means that larger variations on the control action of $u_{2}$ are expected during the transient. It also means that, during steady operation of the plant around a working point, possible perturbations in $y_{1}$ will require a larger control action from $u_{2}$.

Example 10. The second loop is now tuned in order to reduce the perturbation caused by the first loop due to loop interaction. The new controller parameters of the second loop are $k_{c 2}=12$ and $T_{i 2}=2$.

Comparing the new value of $F E T_{c}$ in Eq. (17a) with the previous one from Eq. (16), it can be concluded that the loop interaction is reduced but still large. Using a bandpass filter which selects the frequencies between the open loop and closed loop crossover frequencies results in Eq. (17b). FDPT $T_{c}$ is depicted in Fig. 15. The bandpass filter is represented in Eq. (17a) by $F_{2}$ and it is a $2 \times 2$ transfer function with Butterworth filters of order 2 with a bandpass of [0.0998 rad/s, $2.0052 \mathrm{rad} / \mathrm{s}$ ] for the channels in the
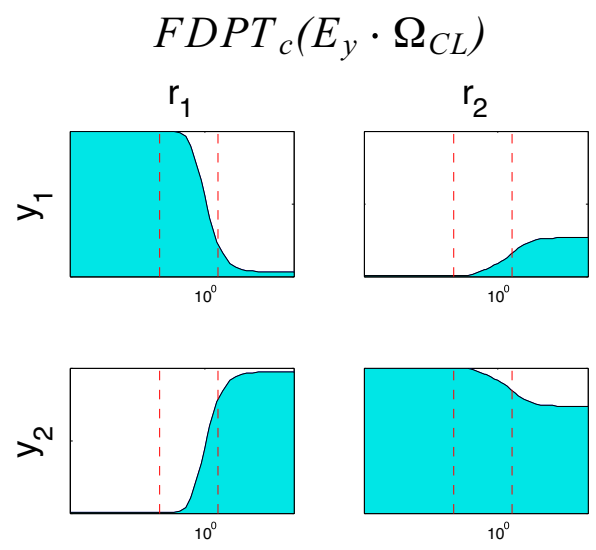

Fig. 15. $F D P T_{c}\left(E_{y} \cdot \boldsymbol{\Omega}_{C L}\right)$ for the closed loop system in Example 9 for frequencies between $10^{-3} \mathrm{rad} / \mathrm{s}$ and $10^{2} \mathrm{rad} / \mathrm{s}$ (logarithmic scales). The ordinates axes are ranged between 0 and 1 (normalized). The dashed lines delimite designed ranges of frequencies for each column which are including the crossover frequencies of the open loop and the closed loop systems. These range of frequencies corresponds with the used for designing the filter $F_{2}$ in Eq. (17b). ProMoVis screenshot. first column and a bandpass of [0.0998 rad/s, $2.5909 \mathrm{rad} / \mathrm{s}]$ for the channels in the second column, and $\otimes$ represents element by element multiplication. It can therefore be concluded that like in the previous controller, the loop interaction will be significant during the transient response in case of a setpoint change, or during steady state in case of the presence of significant process disturbances.

$$
\begin{aligned}
& \left.F E T_{c}\left(E_{y} \cdot \Omega_{C L}\right)=\begin{array}{cc}
r_{1} & r_{2} \\
& 0.1304 \\
0.6897 & 0.8696
\end{array}\right) \mid \begin{array}{l}
y_{1} \\
y_{2}
\end{array} \\
& F E T_{c}\left(F_{2} \otimes\left(E_{y} \cdot \Omega_{C L}\right)\right)=\begin{array}{cc}
r_{1} & r_{2} \\
\left.\hline \begin{array}{ll}
0.4714 & 0.1062 \\
0.5286 & 0.8938
\end{array}\right) & y_{1} \\
y_{2}
\end{array}
\end{aligned}
$$

\section{Conclusions}

The methods presented in [23] to describe the flow of energy in time-invariant processes linearized around a working point have been reformulated in a more general and flexible notation. The new notation is based in signal flow graphs and provides with the additional advantages which include: same formulation for continuous time and discrete systems, possibility of hiding process variables which are not of interest for the analysis, possibility of applying filters in order to restrict the analysis into a desired range of frequencies, and possibility of performing closed loop analysis,. This notation also allowed a more efficient implementation of the methods in the software tool ProMoVis: a tool for Process Modeling and Visualization [15].

An important fact is that the scaling of the signals affects the interpretation of the results [23]. This issue has been solved in this paper by proposing an alternative computation of the methods based on a scaling invariant representation of the linear system.

The validity and usefulness of these methods is reinforced with new examples which successfully determine feasible control structures, and evaluate closed loop behavior.

Applications of the methods in control structure selection have been demonstrated by designing a decentralized control structure for a $2 \times 2$ system, and a sparse control structure for a $5 \times 5$ system. In both cases, the method $F E T_{r}$ with its associated graph, and its counterpart in the frequency domain $F D P T_{r}$ provided useful information for the selection of the control structure. From these analyzed examples and previous experience with similar interaction measures, rules for control structure design using $F E T_{r}$ and $F D P T_{r}$ have been derived.

Using examples, the suggested methods for control structure selection have been compared with other traditional methods including the RGA, and two gramian based IMs: the PM and the $\Sigma_{2}$. A clear advantage of $F E T_{r}$ and $S D P T_{r}$ over the gramian based interaction measures is the insensitivity to output scaling. This is a clear benefit due to the different nature of the process variables which is usually present in industrial processes. This difference involves that the system needs to be properly scaled for the traditional gramian based interaction measures in order to provide trustworthy results. The variables related to the actuators are usually expressed in \% of actuator action, and can in most cases be considered an appropriate scaling.

An additional advantage over the existing interaction measures is that the suggested methods have the potential of being able to analyze if the selected control structure is likely to yield a good rejection of process disturbances. This is done independently for each controlled variable by selecting a set of actuators whose joint contribution on the controlled variable is much larger than the contribution of the process disturbances. However, this proposition still has to be validated with simulations in future research work. 
Once that the controller is designed, the proposed methods can be used to test the performance of the closed loop system. The presented tools can evaluate the effect of changing the references in the controlled variables. A usual requirement for multivariable control systems is the decoupling of the control loops, meaning that for a well designed and well tuned controller, the loop interaction should remain low. This implies that a change in a reference should mainly influence in the response of the controlled variable associated with that reference, being the effect on the other controlled variables low. The tools were also able to identify which of the actuators will require a larger control action to track a change in the reference.

The examples analyzed in this paper are $2 \times 2$ and $5 \times 5$ systems. The application of these methods to large scale systems still needs to be explored. There are several problems which might arise while increasing the number of process variables. A first limitation is a resource limitation, since the methods are model based, and creating models for a process is a time consuming task which increases in complexity when the number of process variables increases. A second limitation is in the visualization of the results, since creating understandable graphs becomes more complicated with the increase in the number of nodes and edges representing the process variables and interconnections. A third limitation is an analysis limitation coming from the fact that many large scale processes usually include transport delays. The insensitivity to time delays of the methods here discussed means that it is not possible with these methods to analyze the influence of time delays on the decision of the control structure to be used. These limitations are subject of future research.

\section{Acknowledgements}

Funding provided by Swedish Governmental Agency for Innovation Systems (VINNOVA) and the Hjalmar Lundbohm Research Center (HLRC) are gratefully acknowledged, as well as the collaboration of the industrial partners of the SCOPE consortium.

\section{Appendix A. Sensitivity of the Hankel norm to time delays}

Assume that a stable SISO system $G(s)$ is excited with an input $u(t)$ up to $t=0$, and the output $u(t)$ is measured for $t>0$. The Hankel norm is then obtained by finding the input $u(t)$ which maximizes:

$\|G(s)\|_{H} \triangleq \max _{u(t)} \frac{\sqrt{\int_{0}^{\infty}\|y(\tau)\|_{2}^{2} d \tau}}{\sqrt{\int_{-\infty}^{0}\|u(\tau)\|_{2}^{2} d \tau}}$

Theorem 1. The Hankel norm of the time delayed system $G_{d}(s)=G(s) \cdot e^{-t_{d}}$ is higher or equal than that of $G(s)$.

Proof. For the same input sequence $u(t), G(s)$ will present an output $y(t)$ and $G_{d}(s)$ will present as output $y\left(t-t_{d}\right)$. And therefore:

$$
\begin{aligned}
\left\|G_{d}(s)\right\|_{H} \triangleq \max _{u(t)} \frac{\sqrt{\int_{0}^{\infty}\left\|y\left(\tau-t_{d}\right)\right\|_{2}^{2} d \tau}}{\sqrt{\int_{-\infty}^{0}\|u(\tau)\|_{2}^{2} d \tau}} \\
\quad=\max _{u(t)} \frac{\sqrt{\int_{-t_{d}}^{0}\|y(\tau)\|_{2}^{2} d \tau+\int_{0}^{\infty}\|y(\tau)\|_{2}^{2} d \tau}}{\sqrt{\int_{-\infty}^{0}\|u(\tau)\|_{2}^{2} d \tau}} \\
\leq \max _{u(t)} \frac{\sqrt{\int_{0}^{\infty}\|y(\tau)\|_{2}^{2} d \tau}}{\sqrt{\int_{-\infty}^{0}\|u(\tau)\|_{2}^{2} d \tau}}
\end{aligned}
$$

\section{References}

[1] M. van de Wal, B. de Jager, A review of methods for input/output selection, Automatica 37 (4) (2001) 487-510.

[2] E. Bristol, On a new measure of interaction for multi-variable process control, IEEE Transactions on Automatic Control 11 (1) (1966) 133-134.

[3] M. Hovd, S. Skogestad, Simple frequency-dependent tools for control systems analysis, structure selection and design, Automatica 28 (5) (1992) 989-996.

[4] V. Manousiouthakis, R. Savage, Y. Arkun, Synthesis of decentralized process control structures using the concept of block relative gain, AIChE Journal 32 (6) (1986) 991-1003.

[5] K.E. Häggblom, Control structure analysis by partial relative gains, in: Proc. of the 36th Conference on Decision \& Control, San Diego, 1997, pp. 2623-2624.

[6] B. Wittenmark, M.E. Salgado, Hankel-norm based interaction measure for input-output pairing, in: Proc. of the 2002 IFAC World Congress, Barcelona, 2002.

[7] M.E. Salgado, A. Conley, Mimo interaction measure and controller structure selection, International Journal of Control 77 (4) (2004) 367-383.

[8] M.E. Sezer, D.D. Šiljak, Nested $\varepsilon$-decompositions and clustering of complex systems, Automatica 22 (3) (1986) 321-331.

[9] A.I.Zečević, D.D. Šiljak, A decomposition-based control strategy for large, sparse dynamic systems, Mathematical Problems in Engineering 1 (2005) 33-48.

[10] S.J. Mason, Feedback theory - some properties of signal flow graphs, in: Proceedings of the IR, 1953, pp. 1144-1156.

[11] W.L. Luyben, B.D. Tyreus, M.L. Luyben, in: Plant-Wide Process Control, McGrawHill, 1998.

[12] S. Skogestad, Control structure design for complete chemical plants, Computers \& Chemical Engineering 28 (1-2) (2004) 219-234.

[13] C. Zhang, S. Vasudevan, G.P. Rangaiah, Plantwide control system design and performance evaluation for ammonia synthesis process, Industrial and Engineering Chemistry Research 49 (24) (2010) 12538-12547.

[14] P. Tofts (Ed.), Quantitative MRI of the Brain: Measuring Changes Caused by Disease, Wiley, Chichester, England, 2003.

[15] W. Birk, A. Johansson, M. Castaño, S. Rönnbäck, T. Nordin, N.-O. Ekholm, Interactive modeling and visualization of complex processes in pulp and paper making, in: Control Systems 2010, Stockholm, 2010.

[16] A. Johansson, Commuting operations on signal-flow graphs for visualization of interconnected systems, in: IFAC Symposium on System, Structure and Control, Ancona, 2010.

[17] S. Skogestad, I. Postlethwaite, in: Multivariable Feedback Control: Analysis and Design, Wiley, New York, 1996

[18] W. Birk, A. Medvedev, A note on gramian-based interaction measures, in: Proc. of the European Control Conference 2003, University of Cambridge, UK, 2003.

[19] K. Johansson, The quadruple-tank process: a multivariable laboratory process with an adjustable zero, IEEE Transactions on Control Systems Technology 8 (3) (2000) 456-465

[20] R. Vadigepalli, E.P. Gatzke, F.J. Doyle, Robust control of a multivariable experimental four-tank system, Industrial and Engineering Chemistry Research 40 (2001) 1916-1927.

[21] A. Khaki-Sedigh, A. Shahmansourian, Input-output pairing using balanced realisations, Electronic Letters 32 (21) (1996) 2027-2028.

[22] B. Halvarsson, Comparison of some gramian based interaction measures, in: IEEE International Conference on Computer-Aided Control Systems, 2008, CACSD 2008, San Antonio, 2008, pp. 138-143.

[23] M. Castaño, W. Birk, New methods for structural and functional analysis of complex processes, in: IEEE International Conference on Computer-Aided Control Systems, 2009, CACSD 2009, Saint Petersburg, 2009, pp. 487-494.

[24] M. Morari, G. Stephanopoulos, Studies in the synthesis of control structures for chemical processes. II. Structural aspects and the synthesis of alternative feasible control schemes, AIChE Journal 26 (2) (1980) 232-246.

[25] T. Steffen, in: Control Reconfiguration of Dynamical Systems, Springer, 2005.

[26] M. Blanke, et al., in: Diagnosis and Fault-Tolerant Control, Springer, 2003.

[27] C. Rech, R. Perret, About structural controllability of interconnected dynamical systems, Automatica 27 (5) (1991) 877-881.

[28] M. Witcher, T. McAvoy, Interacting control systems: steady-state and dynamic measurement of interaction, ISA Transactions 16 (3) (1977) 35-41.

[29] A. Niederlinski, A heuristic approach to the design of linear multivariable interacting control systems, Automatica 7 (1971) 691-701.

[30] Z.-X. Zhu, A. Jutan, Loop decomposition and dynamic interaction analysis of decentralized control systems, Chemical Engineering Science 51 (12) (1996) 3325-3335. 